\title{
Multilayered Scattering Problem with Generalized Impedance Boundary Condition on the Core
}

\author{
Jun Guo, ${ }^{1}$ Guozheng Yan, ${ }^{2}$ and Mingjian $\mathrm{Cai}^{1}$ \\ ${ }^{1}$ School of Mathematics and Statistics, South-Central University for Nationalities, 182 Minyuan Road, Wuhan 430074, China \\ ${ }^{2}$ School of Mathematics and Statistics, Central China Normal University, Wuhan 430079, China \\ Correspondence should be addressed to Jun Guo; hssxgj@126.com
}

Received 29 May 2015; Accepted 3 September 2015

Academic Editor: Carlos J. S. Alves

Copyright (c) 2015 Jun Guo et al. This is an open access article distributed under the Creative Commons Attribution License, which permits unrestricted use, distribution, and reproduction in any medium, provided the original work is properly cited.

\begin{abstract}
This paper is concerned with the scattering problem of time-harmonic acoustic plane waves by an impenetrable obstacle buried in a piecewise homogeneous medium. The so-called generalized impedance boundary condition is imposed on the boundary of the obstacle. Firstly, the well posedness of the solution to the direct scattering problem is established by using the boundary integral method. Then a uniqueness result for the inverse scattering problem is proved; that is, both of the obstacle's shape and the impedances $(\mu, \lambda)$ can be uniquely determined from far field measurements. Furthermore, a mathematical basis is given to reconstruct the shape of the obstacle by using a modified linear sampling method.
\end{abstract}

\section{Introduction}

This work is concerned with the scattering problem of timeharmonic acoustic plane waves by an impenetrable obstacle buried in a piecewise homogeneous medium. We set the generalized impedance boundary condition (GIBC) on the boundary of the obstacle and the transmission boundary conditions on the surface of the layered medium. The GIBC is commonly used to model thin coatings or gratings as well as more accurate models for imperfectly conducting obstacles. Addressing this problem is motivated by applications in nondestructive testing, medical imaging, remote sensing or radar, and so on; at the same time the background may be modeled as a layered medium. For simplicity, we just consider that the unknown obstacle is embedded in a two-layered medium, and the space is $R^{2}$.

To be precise, let $D_{2} \subset R^{2}$ denote the impenetrable obstacle which is a bounded domain with a smooth boundary $S_{1}$ (e.g., $C^{2}$ ). Assume that the unknown obstacle $D_{2}$ is buried in a penetrable obstacle $D$ with a closed $C^{2}$ surface $S_{0}$ such that $\bar{D}_{2} \subset D$. Denote by $D_{1}=D \backslash \bar{D}_{2}$ a connected bounded domain filled with homogeneous medium and denote by $D_{0}=R^{2} \backslash \bar{D}$ the unbounded connected domain occupied by another homogeneous medium. Let $k_{l}=\omega_{l} / c_{l}>0$ be the wave number in terms of the frequency $\omega_{l}$ and the sound speed $c_{l}$ in the corresponding region $D_{l}(l=0,1)$ (see Figure 1).

The scattering of time-harmonic acoustic plane waves by an obstacle with GIBC in a piecewise homogeneous medium in $R^{2}$ can be modeled by the Helmholtz equation with boundary conditions on the boundary $S_{1}$ and interface $S_{0}$ :

$$
\begin{aligned}
\Delta u+k_{0}^{2} u=0, & \text { in } D_{0}, \\
\Delta v+k_{1}^{2} v=0, & \text { in } D_{1}, \\
u_{+}-v_{-}=0, & \text { on } S_{0}, \\
\frac{\partial u_{+}}{\partial v}-\lambda_{0} \frac{\partial v_{-}}{\partial v}=0, & \text { on } S_{0}, \\
\frac{\partial v}{\partial \nu}+\operatorname{div}_{S_{1}}\left(\mu \nabla_{S_{1}} v\right)+\lambda v=0, & \text { on } S_{1} .
\end{aligned}
$$

Here $v$ is the unit outward normal vector on the boundary $S_{0}$ or $S_{1} ; u_{+},\left(\partial u_{+} / \partial v\right)\left(v_{-}, \partial v_{-} / \partial v\right)$ denote the limit of $u$, $(\partial u / \partial v)(v, \partial v / \partial v)$ on the boundary $S_{0}$ from the exterior (interior) of $D$. 


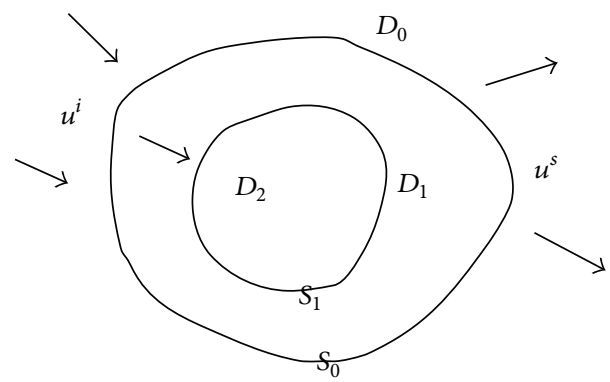

FIGURE 1: Multilayered scattering problem.

Remark 1. In the following discussion, we use “ $(\cdot)_{ \pm}$” or “ $(\cdot)^{ \pm ”}$ to denote the limit approaching the boundary from outside and inside to the corresponding domain, respectively.

The constant surface impedance on $S_{0}$ is supposed $\lambda_{0}>0$ which is given by $\lambda_{0}=\rho_{0} / \rho_{1}$ in terms of the density $\rho_{l}$ in the corresponding region $D_{l}(l=0,1)$. On the boundary $S_{1}$, the impedances $\mu \in C^{1}\left(S_{1}\right)$ and $\lambda \in C\left(S_{1}\right)$ are complex-valued functions satisfying $\operatorname{Im}(\mu) \leq 0$ and $\operatorname{Im}(\lambda) \geq 0$. The surface divergence $\operatorname{div}_{S_{1}}$ and the surface gradient $\nabla_{S_{1}}$ are precisely defined in Chapter 5 of [1]. In the two-dimensional case, the inhomogeneous Laplace-Beltrami differential operator becomes $\operatorname{div}_{S_{1}}\left(\mu \nabla_{S_{1}} v\right)=(d / d s) \mu(d v / d s)$, where $d / d s$ is the tangential derivative and $s$ is the arc length.

The total field $u=u^{s}+u^{i}$ is decomposed into the given incident field $u^{i}=e^{i k_{0} x \cdot d}, d \in \mathbb{S}^{1}$, (the unit sphere in $R^{2}$ ) and the unknown scattered field $u^{s}$ which is required to satisfy the Sommerfeld radiation condition [2]

$$
\lim _{r \rightarrow \infty} \sqrt{r}\left(\frac{\partial u^{s}}{\partial r}-i k_{0} u^{s}\right)=0
$$

uniformly in $\hat{x}=x /|x|$ with $r=|x|$. Further it is known that the scattered field $u^{s}(x, d)$ has the following asymptotic representation:

$$
u^{s}(x, d)=\frac{e^{i k_{0}|x|}}{\sqrt{|x|}}\left\{u^{\infty}(\widehat{x}, d)+O\left(\frac{1}{|x|}\right)\right\}
$$

$$
\text { as }|x| \longrightarrow \infty
$$

uniformly for all directions $\widehat{x}$, where the function $u^{\infty}(\widehat{x}, d)$ defined on the unit sphere $\mathbb{S}^{1}$ is known as the far field pattern with $\widehat{x}$ and $d$ denoting, respectively, the observation direction and the incident direction.

The direct problem is to seek functions $u \in H_{\mathrm{loc}}^{1}\left(D_{0}\right)$ and $v \in H^{1}\left(D_{1}\right)$ satisfying (1) and (2). In the next section, more general direct problem (4) will be considered. If the impenetrable obstacle $D_{2}$ with GIBC is set in a homogeneous medium, it was shown in [3] that there exists a unique solution for the case when the data $h \in H^{-1}\left(S_{1}\right)$ by the variational method; but for the case when $h$ belongs to $H^{-3 / 2}\left(S_{1}\right)$, this method is no longer valid and the difficulty has been resolved in [4] by the integral equation method with the help of the modified Green function technique in [5]. More related works can be found in $[6,7]$. In this paper we will employ the integral equation method to solve direct problem (4) in some Sobolev spaces. The main challenge is to derive a suitable boundary integral system and show that the corresponding boundary integral operators are Fredholm of index zero.

The inverse problem we consider in this paper is to determine the shape of the obstacle $D_{2}$ and $(\mu, \lambda)$ from the knowledge of the far field pattern $u^{\infty}(\widehat{x}, d)$ for all $\hat{x}, d \in \mathbb{S}^{1}$ with the given wave number $k_{l}(l=0,1)$ and the positive constant $\lambda_{0}$.

As usual in most of the inverse problems, the first issue is the uniqueness, that is, in what conditions, the shape of the obstacle $D_{2}$ (or the parameters such as $(\mu, \lambda)$ ) can be uniquely determined by the far field pattern. Through establishing a mixed reciprocity relation, we obtain a uniqueness result in Section 3 (see [6-11] and the references therein).

We solve the above-mentioned inverse problem by using the linear sampling method which was discussed early in 1996 by Colton and Kirsch [12]. The linear sampling method has been developed greatly and applied to solve a variety of inverse problems; we can refer to $[13,14]$ and the references therein. Some other methods also can be used to reconstruct the buried obstacle, for example, the reciprocity gap functional method $[15,16]$ and the Newton iteration method [17].

The remaining part of the paper is organized as follows. In the next section, we will use integral equation method to solve direct scattering problem (4) based on Fredholm theory. In Section 3, we give a uniqueness result, that is, both of the obstacle $D_{2}$ and the impedances $(\mu, \lambda)$ can be uniquely determined from far field measurements. In Section 4 , a mathematical basis is given to reconstruct the shape of the obstacle $D_{2}$ by using a modified linear sampling method.

\section{The Direct Scattering Problem}

In this section, we will establish the well posedness of the direct scattering problem by employing the integral equation method. Let us consider a more general direct scattering problem: Given the transmission boundary conditions $f \in$ $H^{1 / 2}\left(S_{0}\right), g \in H^{-1 / 2}\left(S_{0}\right)$ and a general boundary data $h \in$ $H^{-3 / 2}\left(S_{1}\right)$, find $u \in H_{\mathrm{loc}}^{1}\left(D_{0}\right)$ and $v \in H^{1}\left(D_{1}\right)$ such that

$$
\begin{aligned}
\Delta u+k_{0}^{2} u & =0, & & \text { in } D_{0}, \\
\Delta v+k_{1}^{2} v & =0, & & \text { in } D_{1}, \\
u_{+}-v_{-} & =f, & & \text { on } S_{0}, \\
\frac{\partial u_{+}}{\partial v}-\lambda_{0} \frac{\partial v_{-}}{\partial v} & =g, & & \text { on } S_{0}, \\
\frac{\partial v}{\partial v}+\operatorname{div}_{S_{1}}\left(\mu \nabla_{S_{1}} v\right)+\lambda v & =h, & & \text { on } S_{1}, \\
\lim _{r \rightarrow \infty} \sqrt{r}\left(\frac{\partial u}{\partial r}-i k_{0} u\right) & =0, & & r=|x| .
\end{aligned}
$$

Remark 2. Direct scattering problem (1) and (2) is a special case of problem (4) by taking $u=u^{s}, f=-u^{i}, g=-\partial u^{i} / \partial \nu$, and $h=0$. 
Lemma 3. Suppose that $k>0, \lambda_{0}>0, \operatorname{Im}(\mu) \leq 0$, and $\operatorname{Im}(\lambda) \geq 0$; then problem (4) has at most one solution.

Proof. Clearly, it is sufficient to show that $u=0$ in $D_{0}$ and $v=0$ in $D_{1}$ if $f=g=0$ on $S_{0}$ and $h=0$ on $S_{1}$. Denote by $B_{R}$ a circle large enough with radius $R$ such that $D$ is contained in its interior. From Green's theorem, we obtain

$$
\int_{\partial B_{R}} u \frac{\partial \bar{u}}{\partial \nu} d s=\int_{B_{R} \backslash \bar{D}}\left[|\nabla u|^{2}-k^{2}|u|^{2}\right] d x+\int_{S_{0}} u \frac{\partial \bar{u}}{\partial \nu} d s
$$

in the domain $B_{R} \backslash \bar{D}$ and

$$
\int_{S_{0}} v \frac{\partial \bar{v}}{\partial \nu} d s=\int_{D_{1}}\left[|\nabla v|^{2}-k^{2}|v|^{2}\right] d x+\int_{S_{1}} v \frac{\partial \bar{v}}{\partial \nu} d s
$$

in the domain $D_{1}$. By the boundary conditions of (4), we conclude from the above two equations that

$$
\begin{aligned}
\int_{\partial B_{R}} u \frac{\partial \bar{u}}{\partial \nu} d s= & \int_{B_{R} \mid \bar{D}}\left[|\nabla u|^{2}-k^{2}|u|^{2}\right] d x \\
& +\lambda_{0} \int_{D_{1}}\left[|\nabla v|^{2}-k^{2}|v|^{2}\right] d x \\
& +\lambda_{0} \int_{S_{1}} \bar{\mu}\left|\frac{d v}{d s}\right|^{2} d s-\lambda_{0} \int_{S_{1}} \bar{\lambda}|v|^{2} d s .
\end{aligned}
$$

Since $k>0, \lambda_{0}>0, \operatorname{Im}(\mu) \leq 0$, and $\operatorname{Im}(\lambda) \geq 0$, it follows that

$$
\begin{aligned}
\operatorname{Im}\left(\int_{\partial B_{R}} u \frac{\partial \bar{u}}{\partial \nu} d s\right)= & \lambda_{0} \operatorname{Im}\left(\int_{S_{1}} \bar{\mu}\left|\frac{d u}{d s}\right|^{2} d s\right) \\
& -\lambda_{0} \operatorname{Im}\left(\int_{S_{1}} \bar{\lambda}|u|^{2} d s\right) \geq 0 .
\end{aligned}
$$

Rellich's lemma [2] shows that $u=0$ in $R^{2} \backslash \bar{B}_{R}$ and it follows by the unique continuation principle [2] that $u=0$ in $D_{0}$. The transmission boundary conditions and Holmgren's uniqueness theorem [18] imply that $v=0$ in $D_{1}$. Then we complete the proof of this lemma.

In order to establish the existence of the solution to problem (4), we construct a solution to problem (4) in the form of combined single- and double-layer potentials as follows:

$$
\begin{aligned}
u(x)= & \lambda_{0} \int_{S_{0}} \Phi_{0}(x, y) \varphi(y) d s(y) \\
& +\int_{S_{0}} \frac{\partial \Phi_{0}(x, y)}{\partial \nu(y)} \psi(y) d s(y), \quad x \in D_{0}, \\
v(x)= & \int_{S_{1}} \Phi_{1}(x, y) \eta(y) d s(y) \\
& -\int_{S_{0}} \Phi_{1}(x, y) \varphi(y) d s(y) \\
& -\int_{S_{0}} \frac{\partial \Phi_{1}(x, y)}{\partial \nu(y)} \psi(y) d s(y), \quad x \in D_{1},
\end{aligned}
$$

where $\varphi \in H^{-1 / 2}\left(S_{0}\right), \psi \in H^{1 / 2}\left(S_{0}\right)$, and $\eta \in H^{-1 / 2}\left(S_{1}\right)$ are the unknown densities and

$$
\Phi_{l}:=\frac{i}{4} H_{0}^{(1)}\left(k_{l}|x-y|\right), \quad x \neq y,
$$

$l=0,1$, is the fundamental solution of the Helmholtz equation in $R^{2}$.

Remark 4. Based on the method proposed in [19] for the transmission problem and in [4] for the obstacle scattering with GIBC, we choose the solution as the form of (9). As the authors in [4] point out that the obtained integral equation fails to be uniquely solvable if the irregular frequencies occur. In order to exclude the irregular frequencies, we make the following assumption.

Assumption A. $k_{1}^{2}$ is not a Dirichlet eigenvalue of $-\Delta$ operator in the domain $D_{2}$ which can guarantee the well posedness of the direct problem.

For further consideration, we define the single- and double-layer operators $S_{i j . l}$ and $K_{i j . l}$, respectively, by

$$
\begin{aligned}
\left(S_{i j . l} \varphi\right)(x):=\int_{S_{i}} \varphi(y) \Phi_{l}(x, y) d s(y), \quad x \in S_{j}, \\
\left(K_{i j . l} \varphi\right)(x):=\int_{S_{i}} \varphi(y) \frac{\Phi_{l}(x, y)}{\partial \nu(y)} d s(y), \quad x \in S_{j}
\end{aligned}
$$

and the normal derivative operators $K_{i j . l}^{\prime}$ and $T_{i j . l}$ by

$$
\begin{array}{r}
\left(K_{i j . l}^{\prime} \varphi\right)(x):=\int_{S_{i}} \varphi(y) \frac{\partial \Phi_{l}(x, y)}{\partial \nu(x)} d s(y), \quad x \in S_{j}, \\
\left(T_{i j . l} \varphi\right)(x):=\frac{\partial}{\partial \nu(x)} \int_{S_{i}} \varphi(y) \frac{\partial \Phi_{l}(x, y)}{\partial \nu(y)} d s(y), \\
x \in S_{j},
\end{array}
$$

with $i, j, l=0,1$. Referring to [20], we have mapping properties

$$
\begin{gathered}
S_{i j . l}: H^{-1 / 2+s}\left(S_{i}\right) \longrightarrow H^{1 / 2+s}\left(S_{j}\right), \\
K_{i j . l}: H^{1 / 2+s}\left(S_{i}\right) \longrightarrow H^{1 / 2+s}\left(S_{j}\right), \\
K_{i j . l}^{\prime}: H^{-1 / 2+s}\left(S_{i}\right) \longrightarrow H^{-1 / 2+s}\left(S_{j}\right), \\
T_{i j . l}: H^{1 / 2+s}\left(S_{i}\right) \longrightarrow H^{-1 / 2+s}\left(S_{j}\right),
\end{gathered}
$$

for $i, j, l=0,1$ and $-1 \leq s \leq 1$.

Now we try to establish an integral system by employing the boundary integral equation approach. According to the presentation of the solution in the form of (9) and by making 
use of the known jump relations of single- and double-layer potentials [19], we have that on the interface $S_{0}$

$$
\begin{aligned}
\left.\left(u_{+}-v_{-}\right)\right|_{S_{0}}= & \left(\lambda_{0} S_{00.0}+S_{00.1}\right) \varphi \\
& +\left(K_{00.0}+K_{00.1}\right) \psi-S_{10.1} \eta \\
\left.\left(\frac{\partial u_{+}}{\partial \nu}-\lambda_{0} \frac{\partial v_{-}}{\partial \nu}\right)\right|_{S_{0}}= & \lambda_{0}\left(K_{00.0}^{\prime}+K_{00.1}^{\prime}\right) \varphi \\
& +\left(T_{00.0}+\lambda_{0} T_{00.1}\right) \psi \\
& -\lambda_{0} K_{10.1}^{\prime} \eta
\end{aligned}
$$

On the boundary $S_{1}$, we obtain that

$$
\begin{gathered}
\left.\left(\frac{\partial v}{\partial v}+\operatorname{div}_{S_{1}}\left(\mu \nabla_{S_{1}} v\right)+\lambda v\right)\right|_{S_{1}} \\
=\frac{d}{d s} \mu \frac{d}{d s} S_{11.1} \eta-\frac{1}{2} \eta+K_{11.1}^{\prime} \eta+\lambda S_{11.1} \eta \\
\quad-\frac{d}{d s} \mu \frac{d}{d s} S_{01.1} \varphi-K_{01.1}^{\prime} \varphi-\lambda S_{01.1} \varphi \\
-\frac{d}{d s} \mu \frac{d}{d s} K_{01.1} \psi-T_{01.1} \psi-\lambda K_{01.1} \psi
\end{gathered}
$$

Define bounded linear operators $A_{11}, B_{11}: H^{-1 / 2}\left(S_{1}\right) \rightarrow$ $H^{-3 / 2}\left(S_{1}\right)$ by

$$
\begin{aligned}
A_{11.1} \eta:= & \mu \frac{d^{2}}{d s^{2}} S_{11.1} \eta+\mu \int_{S_{1}} S_{11.1} \eta d s \\
B_{11} \eta:= & \frac{d \mu}{d s} \frac{d}{d s} S_{11.1} \eta-\frac{1}{2} \eta+K_{11.1}^{\prime} \eta+\lambda S_{11.1} \eta \\
& -\mu \int_{S_{1}} S_{11.1} \eta d s .
\end{aligned}
$$

Let

$$
\begin{aligned}
& M=\frac{d}{d s} \mu \frac{d}{d s} S_{01.1} \varphi+K_{01.1}^{\prime} \varphi+\lambda S_{01.1} \varphi, \\
& N=\frac{d}{d s} \mu \frac{d}{d s} K_{01.1} \psi+T_{01.1} \psi+\lambda K_{01.1} \psi, \\
& \omega=\left(\begin{array}{c}
\varphi \\
\psi \\
\eta
\end{array}\right), \\
& R=\left(\begin{array}{c}
f \\
-\frac{g}{\lambda_{0}} \\
h
\end{array}\right),
\end{aligned}
$$

A

$$
=\left(\begin{array}{ccc}
\lambda_{0} S_{00.0}+S_{00.1} & K_{00.0}+K_{00.1} & -S_{10.1} \\
-K_{00.0}^{\prime}-K_{00.1}^{\prime} & -\frac{1}{\lambda_{0}} T_{00.0}-T_{00.1} & K_{10.1}^{\prime} \\
-M & -N & A_{11}+B_{11}
\end{array}\right) .
$$

Then the potential functions defined by (9) solve problem (4) provided the unknown densities $\varphi, \psi$, and $\eta$ solve the following boundary integral system:

$$
A \omega=R
$$

Defining the Sobolev spaces

$$
\begin{aligned}
& H=H^{-1 / 2}\left(S_{0}\right) \times H^{1 / 2}\left(S_{0}\right) \times H^{-1 / 2}\left(S_{1}\right), \\
& W=H^{1 / 2}\left(S_{0}\right) \times H^{-1 / 2}\left(S_{0}\right) \times H^{-3 / 2}\left(S_{1}\right),
\end{aligned}
$$

it is easy to see that the matrix operator $A$ maps $H$ continuously into $W$.

Based on the following two lemmas, we show the solvability of (18) by using the Fredholm theory.

Lemma 5. The operator A given by (18) is Fredholm with index zero.

Proof. From [20], the operators $S_{00.0}, S_{00.1},-T_{00.0}$, and $-T_{00.1}$ are positive and bounded up to a compact perturbation, respectively; we denote by $L_{0}, L_{1}, J_{0}$, and $J_{1}$ the compact operators

$$
\begin{gathered}
L_{l}: H^{-1 / 2}\left(S_{0}\right) \longrightarrow H^{1 / 2}\left(S_{0}\right) ; \\
J_{l}: H^{1 / 2}\left(S_{0}\right) \longrightarrow H^{-1 / 2}\left(S_{0}\right),
\end{gathered}
$$

$$
l=0,1
$$

such that

$$
\begin{gathered}
\operatorname{Re}\left(\left\langle\left(S_{00 . l}+L_{l}\right) \varphi, \bar{\varphi}\right\rangle\right) \geq C\|\varphi\|_{H^{-1 / 2}\left(S_{0}\right)}^{2}, \\
\text { for } \varphi \in H^{-1 / 2}\left(S_{0}\right), \\
\operatorname{Re}\left(\left\langle-\left(T_{00 . l}+J_{l}\right) \psi, \bar{\psi}\right\rangle\right) \geq C\|\psi\|_{H^{1 / 2}\left(S_{0}\right)}^{2}, \\
\text { for } \psi \in H^{1 / 2}\left(S_{0}\right),
\end{gathered}
$$

where $\langle$,$\rangle denotes the duality between H^{-1 / 2}\left(S_{0}\right)$ and $H^{1 / 2}\left(S_{0}\right)$.

Let $K$ and $K^{\prime}$ be the operators defined as $K_{00.0}$ and $K_{00.0}^{\prime}$, respectively, with kernel $\Phi_{0}(x, y)$ replaced by $\Phi(x, y)=$ $-(1 / 2 \pi) \ln |x-y|$. Then $K_{l c}=K_{00 . l}-K$ and $K_{l c}^{\prime}=K_{00 . l}^{\prime}-$ $K^{\prime}(l=0,1)$ are compact since they have continuous kernels. It is easy to show that $K$ and $K^{\prime}$ are adjoint since their kernels are real; that is,

$$
(K \psi, \phi)=\left(\psi, K^{\prime} \phi\right)
$$


Now, we decompose $A$ into two parts; that is,

$$
\begin{aligned}
A= & \left(\begin{array}{ccc}
\lambda_{0} S^{0}+S^{1} & 2 K & 0 \\
-2 K^{\prime} & -\frac{1}{\lambda_{0}} T^{0}-T^{1} & 0 \\
0 & 0 & A_{11}
\end{array}\right) \\
& +\left(\begin{array}{ccc}
-\lambda_{0} L_{0}-L_{1} & K_{0 c}+K_{1 c} & -S_{10.1} \\
-K_{0 c}^{\prime}-K_{1 c}^{\prime} & \frac{1}{\lambda_{0}} J_{0}+J_{1} & K_{10.1}^{\prime} \\
-M & -N & B_{11}
\end{array}\right) \\
:= & A_{0}+A_{c},
\end{aligned}
$$

where $S^{l}=S_{00 . l}+L_{l}, T^{l}=T_{00 . l}+J_{l}$ for $(l=0,1)$. Consider the following sesquilinear form for $(\varphi, \psi)^{\top} \in U:=H^{-1 / 2}\left(S_{0}\right) \times$ $H^{1 / 2}\left(S_{0}\right)$ :

$$
\begin{gathered}
\left\langle\left(\begin{array}{cc}
\lambda_{0} S^{0}+S^{1} & 2 K \\
-2 K^{\prime} & -\frac{1}{\lambda_{0}} T^{0}-T^{1}
\end{array}\right)\left(\begin{array}{c}
\varphi \\
\psi
\end{array}\right),\left(\begin{array}{c}
\bar{\varphi} \\
\bar{\psi}
\end{array}\right)\right\rangle_{U^{*}, U} \\
=\lambda_{0}\left(S^{0} \varphi, \varphi\right)+\left(S^{1} \varphi, \varphi\right)+\frac{1}{\lambda_{0}}\left(-T^{0} \psi, \psi\right) \\
+\left(-T^{1} \psi, \psi\right)+2(K \psi, \varphi)-2\left(K^{\prime} \varphi, \psi\right),
\end{gathered}
$$

where $U^{*}$ is the dual space of $U$ and $(\cdot, \cdot)$ denotes the scalar product on $L^{2}\left(S_{0}\right)$. Due to the coercivity of $S^{l}$ and $-T^{l}$, the adjoint between $K$ and $K^{\prime}$, we obtain that the above sesquilinear form is coercive; that is,

$$
\begin{aligned}
& \operatorname{Re}\left\langle\left(\begin{array}{cc}
\lambda_{0} S^{0}+S^{1} & 2 K \\
-2 K^{\prime} & -\frac{1}{\lambda_{0}} T^{0}-T^{1}
\end{array}\right)\left(\begin{array}{c}
\varphi \\
\psi
\end{array}\right),\right. \\
& \left.\left(\begin{array}{c}
\bar{\varphi} \\
\bar{\psi}
\end{array}\right)\right\rangle_{U^{*}, U} \geq C\left(\|\varphi\|_{\mathrm{H}^{-1 / 2}\left(S_{0}\right)}+\|\psi\|_{H^{1 / 2}\left(S_{0}\right)}\right) .
\end{aligned}
$$

Whence the operator

$$
\left(\begin{array}{cc}
\lambda_{0} S^{0}+S^{1} & 2 K \\
-2 K^{\prime} & -\frac{1}{\lambda_{0}} T^{0}-T^{1}
\end{array}\right): U \longrightarrow U^{*}
$$

is invertible. On the other hand, by our Assumption $A$, it can be seen that $A_{11}: H^{-1 / 2}\left(S_{1}\right) \rightarrow H^{-3 / 2}\left(S_{1}\right)$ is invertible (see Lemma 2.1 in [21]). So the operator $A_{0}$ is invertible.

The entries $S_{10.1}, K_{10.1}^{\prime}, M$, and $N$ have continuous kernels, which means that they are compact operators. Due to the compact embedding theorem and the mapping properties of $S_{11.1}$ and $K_{11.1}^{\prime}$, the entry $B_{11}$ is compact. As stated above, the other entries are also compact. We conclude that $A_{c}$ is compact. So we complete the proof of this lemma.

Lemma 6. The operator A given by (18) has a trivial kernel.
Proof. Let $\xi=(\alpha, \beta, \delta)^{\top} \in H$ satisfying $A \xi=\mathbf{0}$. Define two potentials

$$
\begin{aligned}
q(x)= & \lambda_{0} \int_{S_{0}} \Phi_{0}(x, y) \alpha(y) d s(y) \\
& +\int_{S_{0}} \frac{\partial \Phi_{0}(x, y)}{\partial \nu(y)} \beta(y) d s(y), \quad x \in D, \\
q(x)= & \int_{S_{1}} \Phi_{1}(x, y) \delta(y) d s(y) \\
& -\int_{S_{0}} \Phi_{1}(x, y) \alpha(y) d s(y) \\
& -\int_{S_{0}} \frac{\partial \Phi_{1}(x, y)}{\partial \nu(y)} \beta(y) d s(y), \quad x \in D_{0} .
\end{aligned}
$$

Using the jump relations of the single- and double-layer potentials across $S_{0}$, we have

$$
\begin{aligned}
\left.\left(q_{-}-q_{+}\right)\right|_{S_{0}}= & \left(\lambda_{0} S_{00.0}+S_{00.1}\right) \alpha \\
& +\left(K_{00.0}+K_{00.1}\right) \beta-S_{10.1} \eta \\
\left.\left(\frac{\partial q_{-}}{\partial \nu}-\lambda_{0} \frac{\partial q_{+}}{\partial \nu}\right)\right|_{S_{0}}= & \lambda_{0}\left(K_{00.0}^{\prime}+K_{00.1}^{\prime}\right) \alpha \\
& +\left(T_{00.0}+\lambda_{0} T_{00.1}\right) \beta \\
& -\lambda_{0} K_{10.1}^{\prime} \delta .
\end{aligned}
$$

Since $A \xi=\mathbf{0}$, it is easy to check that the potentials defined in (27) and (28) satisfy

$$
\begin{aligned}
\Delta q+k_{1}^{2} q=0, & \text { in } D_{0}, \\
\Delta q+k_{0}^{2} q=0, & \text { in } D, \\
q_{+}-q_{-}=0, & \text { on } S_{0}, \\
\lambda_{0} \frac{\partial q_{+}}{\partial v}-\frac{\partial q_{-}}{\partial v} & =0, \quad \text { on } S_{0}, \\
\lim _{r \rightarrow \infty} \sqrt{r}\left(\frac{\partial q}{\partial r}-i k_{1} q\right) & =0, \quad r=|x| .
\end{aligned}
$$

We can show that problem (30) has only trivial solution (see [2]).

Using the same $(\alpha, \beta, \delta)^{\top}$, define two new potentials

$$
\begin{aligned}
p(x)= & \lambda_{0} \int_{S_{0}} \Phi_{0}(x, y) \alpha(y) d s(y) \\
& +\int_{S_{0}} \frac{\partial \Phi_{0}(x, y)}{\partial \nu(y)} \beta(y) d s(y), \quad x \in D_{0},
\end{aligned}
$$




$$
\begin{aligned}
p(x)= & \int_{S_{1}} \Phi_{1}(x, y) \delta(y) d s(y) \\
& -\int_{S_{0}} \Phi_{1}(x, y) \alpha(y) d s(y) \\
& -\int_{S_{0}} \frac{\partial \Phi_{1}(x, y)}{\partial \nu(y)} \beta(y) d s(y), \quad x \in D_{1} .
\end{aligned}
$$

Then we can prove that $p$ satisfies problem (4) with homogeneous boundary conditions. Lemma 3 shows that $p=0$. Thus again by the jump relations of the single- and doublelayer potentials across $S_{0}$ we have

$$
\begin{gathered}
\beta=\left.\left(p_{+}-q_{-}\right)\right|_{S_{0}}=0, \\
\lambda_{0} \alpha=\left.\left(\frac{\partial q_{-}}{\partial \nu}-\frac{\partial p_{+}}{\partial \nu}\right)\right|_{S_{0}}=0 .
\end{gathered}
$$

At this time, the potential given by (28) becomes

$$
q(x)=\int_{S_{1}} \Phi_{1}(x, y) \delta(y) d s(y),
$$

and note that $q=0$ on $S_{1}$ because of the trivial solution of (30); we conclude that $q$ satisfies the Helmholtz equation in $D_{2}$ with homogeneous Dirichlet boundary condition if we let $x \in D_{2}$. By our Assumption $A, k_{1}^{2}$ is not a Dirichlet eigenvalue in $D_{2}$, which implies that $q=0$ in $D_{2}$. Therefore, the jump relation across $S_{1}$ shows that

$$
\delta=\left.\left(\frac{\partial q_{-}}{\partial \nu}-\frac{\partial p_{+}}{\partial v}\right)\right|_{S_{1}}=0 .
$$

Then we complete the proof of this lemma.

By Fredholm theory, the above two lemmas show that the matrix operator $A$ given by (18) has a bounded inverse; as a consequence, we have the following.

Theorem 7. Under Assumption A, integral system (18) has a unique solution, and problem (4) has a unique solution given by (9) which satisfies

$$
\begin{aligned}
& \|u\|_{H_{l o c}^{1}\left(D_{0}\right)}+\|v\|_{H^{1}\left(D_{1}\right)} \\
& \quad \leq C\left(\|f\|_{H^{1 / 2}\left(S_{0}\right)}+\|g\|_{H^{-1 / 2}\left(S_{0}\right)}+\|h\|_{H^{-3 / 2}\left(S_{1}\right)}\right) .
\end{aligned}
$$

\section{A Uniqueness Result of the Inverse Problem}

As usual in most of the inverse problems, the first question to ask is the identifiability, that is, whether the scatterer $D_{2}$ and $(\mu, \lambda)$ can be identified from a knowledge of the far field pattern. Mathematically, the identifiability is the uniqueness issue which is of theoretical interest and is required in order to proceed to efficient numerical methods of solutions.

Let us go back to scattering problem (1) and (2). The incident wave has two choices: the incident plane wave $u^{i}(\cdot, d)$ (i.e., $u^{i}(x, d)=e^{i k x \cdot d}$ ) and the incident point source $\Phi(\cdot, z)$.
(1) To the incident plane wave $u^{i}(\cdot, d)$, we use $u^{s}(\cdot, d)$ and $u^{\infty}(\cdot, d)$ to denote the scattered field and the corresponding far field pattern, respectively.

(2) To the incident point source $\Phi(\cdot, z)$, we use $u^{s}(\cdot, z)$ and $\Phi^{\infty}(\cdot, z)$ to denote the scattered field and the corresponding far field pattern, respectively.

The uniqueness result is based on the following mixed reciprocity relation.

Lemma 8. For the scattering of plane waves $u^{i}(\cdot, d)$ with $d \epsilon$ $\mathbb{S}^{1}$ and point sources $\Phi_{0}(\cdot, z)$, we have

$$
\begin{aligned}
& \Phi_{0}^{\infty}(\widehat{x}, z) \\
& = \begin{cases}\gamma u^{s}(z,-\widehat{x}) & z \in D_{0}, \hat{x} \in \mathbb{S}^{1}, \\
\lambda_{0} \gamma u^{s}(z,-\hat{x})+\left(\lambda_{0}-1\right) \gamma u^{i}(z,-\hat{x}) & z \in D_{1}, \hat{x} \in \mathbb{S}^{1},\end{cases}
\end{aligned}
$$

where $\gamma=e^{i \pi / 4} / \sqrt{8 k_{0} \pi}$.

Remark 9. The mixed reciprocity relation has been established in the case of obstacle scattering problem $[7,11,18]$; here we extend the result to the scattering problem by an obstacle with GIBC buried in a piecewise homogeneous medium.

Proof. We consider the case $z \in D_{0}$ firstly. By Green's second theorem and the Sommerfeld radiation condition we have that

$$
\begin{aligned}
\int_{S_{0}} & \left(u_{+}^{s}(y, z) \frac{\partial u_{+}^{s}(y, d)}{\partial \nu(y)}\right. \\
& \left.-\frac{\partial u_{+}^{s}(y, z)}{\partial \nu(y)} u_{+}^{s}(y, d)\right) d s(y)=0
\end{aligned}
$$

for $z \in D_{0}, d \in \mathbb{S}^{1}$. By the boundary conditions and Green's second theorem, the total fields $u(\cdot, d)=u^{s}(\cdot, d)+u^{i}(\cdot, d)$ and $u(\cdot, z)=u^{s}(\cdot, z)+\Phi(\cdot, z)$ satisfy

$$
\begin{aligned}
\int_{S_{0}} & \left(u_{+}(y, z) \frac{\partial u_{+}(y, d)}{\partial \nu(y)}\right. \\
- & \left.\frac{\partial u_{+}(y, z)}{\partial \nu(y)} u_{+}(y, d)\right) d s(y) \\
= & \lambda_{0} \int_{S_{0}}\left(v_{-}(y, z) \frac{\partial v_{-}(y, d)}{\partial v(y)}\right. \\
- & \left.\frac{\partial v_{-}(y, z)}{\partial v(y)} v_{-}(y, d)\right) d s(y) \\
= & \lambda_{0} \int_{S_{1}}\left(\mu \nabla_{S_{1}} v(\cdot, z) \cdot \nabla_{S_{1}} v(\cdot, d)\right. \\
- & \lambda v(\cdot, z) v(\cdot, d)) d s-\lambda_{0} \int_{S_{1}}\left(\mu \nabla_{S_{1}} v(\cdot, d)\right. \\
\cdot & \left.\nabla_{S_{1}} v(\cdot, z)+\lambda v(\cdot, d) v(\cdot, z)\right) d s=0 .
\end{aligned}
$$


Since the incident plane wave $u^{i}(\cdot, d)$ and incident point source $\Phi_{0}(\cdot, z)$ solve the Helmholtz equation inside $D$, we obtain that from the above two equalities

$$
\begin{gathered}
\int_{S_{0}}\left(u_{+}^{s}(y, z) \frac{\partial u^{i}(y, d)}{\partial \nu(y)}\right. \\
\left.-\frac{\partial u_{+}^{s}(y, z)}{\partial \nu(y)} u^{i}(y, d)\right) d s(y) \\
=\int_{S_{0}}\left(u_{+}^{s}(y, d) \frac{\partial \Phi_{0}(y, z)}{\partial \nu(y)}\right. \\
\left.-\frac{\partial u_{+}^{s}(y, d)}{\partial \nu(y)} \Phi_{0}(y, z)\right) d s(y) .
\end{gathered}
$$

Using Green's representation formula for $u^{s}(\cdot, \widehat{x})$, we obtain the representation

$$
\begin{gathered}
u^{s}(z, \widehat{x})=\int_{S_{0}}\left(u_{+}^{s}(y, \widehat{x}) \frac{\partial \Phi_{0}(y, z)}{\partial \nu(y)}\right. \\
\left.-\frac{\partial u_{+}^{s}(y, \widehat{x})}{\partial \nu(y)} \Phi_{0}(y, z)\right) d s(y)
\end{gathered}
$$

for $\widehat{x} \in \mathbb{S}^{1}$. The far field pattern $\Phi_{0}^{\infty}(\widehat{x}, z)$ has the following integral representation:

$$
\begin{gathered}
\Phi_{0}^{\infty}(-\widehat{x}, z)=\gamma \int_{S_{0}}\left(u_{+}^{s}(y, z) \frac{\partial u^{i}(y, \widehat{x})}{\partial \nu(y)}\right. \\
\left.-\frac{\partial u_{+}^{s}(y, z)}{\partial \nu(y)} u^{i}(y, \widehat{x})\right) d s(y)
\end{gathered}
$$

Thus we conclude that $\Phi_{0}^{\infty}(-\widehat{x}, z)=\gamma u^{s}(z, \widehat{x})$ for $z \in D_{0}$, $\hat{x} \in \mathbb{S}^{1}$ from (39), (40), and (41) with $d$ replaced by $\hat{x}$.

Next, we consider the case $z \in D_{1}$. From the boundary condition on $S_{1}$, we have that for the total fields $v(\cdot, d)$ and $v(\cdot, z)$

$$
\begin{aligned}
& \int_{S_{1}}\left(v(y, z) \frac{\partial v(y, d)}{\partial v(y)}-\frac{\partial v(y, z)}{\partial v(y)} v(y, d)\right) d s(y) \\
& \quad=0
\end{aligned}
$$

For the scattered fields $u^{s}(\cdot, d)$ and $u^{s}(\cdot, z)$ we still have equality (37), and for the incident plane wave $u^{i}(\cdot, d)$ and incident point source $\Phi_{0}(\cdot, z)$ we have

$$
\begin{aligned}
\int_{S_{1}}\left(u^{i}(y, d) \frac{\partial \Phi_{0}(y, z)}{\partial \nu(y)}\right. \\
\left.\quad-\frac{\partial u^{i}(y, d)}{\partial \nu(y)} \Phi_{0}(y, z)\right) d s(y)=0
\end{aligned}
$$

by Green's second theorem.
From (37) and (41) we get

$$
\begin{gathered}
\Phi_{0}^{\infty}(-\hat{x}, z)=\gamma \int_{S_{0}}\left(u_{+}^{s}(y, z) \frac{\partial u_{+}(y, \widehat{x})}{\partial \nu(y)}\right. \\
\left.-u_{+}(y, \widehat{x}) \frac{\partial u_{+}^{s}(y, z)}{\partial \nu(y)}\right) d s(y)
\end{gathered}
$$

Let $S(z ; \epsilon):=\left\{y \in R^{2}:|y-z|=\epsilon\right\}$ be a sphere contained in $D_{1}$. Applying Green's second theorem in the domain $B(z ; \epsilon):=\left\{y \in D_{1}:|y-z|>\epsilon\right\}$ and taking into account the boundary condition on $S_{0}$ we further have

$$
\begin{aligned}
& \Phi_{0}^{\infty}(-\widehat{x}, z)=\gamma \lambda_{0} \int_{S_{1}}\left(u^{s}(y, z) \frac{\partial v(y, \widehat{x})}{\partial \nu(y)}\right. \\
& \left.-v(y, \widehat{x}) \frac{\partial u^{s}(y, z)}{\partial \nu(y)}\right) d s(y) \\
& +\gamma \lambda_{0} \int_{S(z ; \epsilon)}\left(u^{s}(y, z) \frac{\partial v(y, \widehat{x})}{\partial \nu(y)}\right. \\
& \left.-v(y, \widehat{x}) \frac{\partial u^{s}(y, z)}{\partial \nu(y)}\right) d s(y) \\
& +\gamma \lambda_{0} \int_{B(z ; \epsilon)}\left(k_{1}^{2}-k_{0}^{2}\right) \Phi_{0}(y, z) v(y, \widehat{x}) d y+(1 \\
& \left.-\lambda_{0}\right) \gamma \int_{S_{0}} v_{-}(y, \widehat{x}) \frac{\partial \Phi_{0}(y, z)}{\partial \nu(y)} d s(y) .
\end{aligned}
$$

By the well posedness of the direct problem and the interior elliptic regularity [22], $v(\cdot, \widehat{x}) \in C^{\infty}\left(D_{1}\right)$ and $u^{s}(\cdot, z) \in H^{2}(V)$ for any compact subset $V$ of $D_{1}$. Therefore, there is a sequence $\epsilon_{j}$ such that $\epsilon_{j} \rightarrow 0$ and

$$
\int_{S\left(z ; \epsilon_{j}\right)}\left(\left|u^{s}(y, z)\right|^{2}+\left|\nabla u^{s}(y, z)\right|^{2}\right) d s(y) \longrightarrow 0
$$

as $j \rightarrow \infty$. This together with the Cauchy-Schwarz inequality implies that the integral on $S\left(z ; \epsilon_{j}\right)$ tends to 0 as $j \rightarrow \infty$. By passing to the limit $j \rightarrow \infty$ in (45) with $\epsilon=\epsilon_{j}$ we have

$$
\begin{gathered}
\Phi_{0}^{\infty}(-\widehat{x}, z)=\gamma \lambda_{0} \int_{S_{1}}\left(u^{s}(y, z) \frac{\partial v(y, \widehat{x})}{\partial \nu(y)}\right. \\
\left.-v(y, \widehat{x}) \frac{\partial u^{s}(y, z)}{\partial \nu(y)}\right) d s(y)+\gamma \lambda_{0} \int_{D_{1}}\left(k_{1}^{2}\right. \\
\left.-k_{0}^{2}\right) \Phi_{0}(y, z) v(y, \widehat{x}) d y+\left(1-\lambda_{0}\right) \\
\cdot \gamma \int_{S_{0}} v_{-}(y, \widehat{x}) \frac{\partial \Phi_{0}(y, z)}{\partial v(y)} d s(y) .
\end{gathered}
$$

The volume integral exists as an improper integral since its integrand is weakly singular. 
On the other hand, by Green's representation formula and Green's second theorem, we have that

$$
\begin{aligned}
& \gamma \lambda_{0} v(z, \widehat{x})=\gamma \lambda_{0} \int_{S_{0}}\left(\Phi_{0}(z, y) \frac{\partial v_{-}(y, \hat{x})}{\partial \nu(y)}\right. \\
& \left.-v_{-}(y, \widehat{x}) \frac{\partial \Phi_{0}(z, y)}{\partial \nu(y)}\right) d s(y) \\
& -\gamma \lambda_{0} \int_{S_{1}}\left(\Phi_{0}(z, y) \frac{\partial v(y, \widehat{x})}{\partial \nu(y)}\right. \\
& \left.-v(y, \widehat{x}) \frac{\partial \Phi_{0}(z, y)}{\partial v(y)}\right) d s(y)+\gamma \lambda_{0} \int_{D_{1}}\left(k_{1}^{2}\right. \\
& \left.-k_{0}^{2}\right) \Phi_{0}(z, y) v(y, \widehat{x}) d y .
\end{aligned}
$$

It follows from (47) and (48) together with the boundary conditions on $S_{0}$ and $S_{1}$ and Green's second theorem that

$$
\begin{aligned}
& \Phi_{0}^{\infty}(-\widehat{x}, z)-\gamma \lambda_{0} v(z, \widehat{x})=\left(1-\lambda_{0}\right) \gamma \int_{S_{0}} \nu_{-}(y, \widehat{x}) \\
& \frac{\partial \Phi_{0}(y, z)}{\partial \nu(y)} d s(y) \\
& -\gamma \lambda_{0} \int_{S_{0}}\left(\Phi_{0}(z, y) \frac{\partial \nu_{-}(y, \widehat{x})}{\partial \nu(y)}\right. \\
& \left.-v_{-}(y, \widehat{x}) \frac{\partial \Phi_{0}(z, y)}{\partial \nu(y)}\right) d s(y) \\
& =\gamma \int_{S_{0}}\left(u_{+}(y, \hat{x}) \frac{\partial \Phi_{0}(z, y)}{\partial \nu(y)}\right. \\
& \left.-\Phi_{0}(z, y) \frac{\partial u_{+}(y, \hat{x})}{\partial \nu(y)}\right) d s(y) \\
& =\gamma \int_{S_{0}}\left(u^{i}(y, \widehat{x}) \frac{\partial \Phi_{0}(z, y)}{\partial \nu(y)}\right. \\
& \left.-\Phi_{0}(z, y) \frac{\partial u^{i}(y, \hat{x})}{\partial \nu(y)}\right) d s(y) \\
& +\gamma \int_{S_{0}}\left(u_{+}^{s}(y, \widehat{x}) \frac{\partial \Phi_{0}(z, y)}{\partial \nu(y)}\right. \\
& \left.-\Phi_{0}(z, y) \frac{\partial u_{+}^{s}(y, \widehat{x})}{\partial \nu(y)}\right) d s(y)=-\gamma u^{i}(z, \widehat{x}) .
\end{aligned}
$$

We conclude from (49) that $\Phi_{0}^{\infty}(-\hat{x}, z)=\lambda_{0} \gamma u^{s}(z, \hat{x})+\left(\lambda_{0}-\right.$ 1) $\gamma u^{i}(z, \hat{x})$, for $z \in D_{1}, \widehat{x} \in \mathbb{S}^{1}$. Therefore the proof of this lemma is completed.

Lemma 10. For the transmitted wave $v$ of problem (1) and (2) associated with the incident plane wave $u^{i}=e^{i k_{0} x \cdot d}$, we have that $\mathbb{V}=\left\{\left.v(\cdot, d)\right|_{S_{1}}, d \in \mathbb{S}^{1}\right\}$ is complete in $H^{3 / 2}\left(S_{1}\right)$.
Note that for the incident plane wave $u^{i}$, the regularity of elliptic equations shows that the solution of problem (1) and (2) belongs to $H_{\text {loc }}^{2}\left(D_{0}\right) \cap H^{2}\left(D_{1}\right)$.

Proof. Assume that $\phi$ is a function in $H^{-3 / 2}\left(S_{1}\right)$ such that for every $d \in \mathbb{S}^{1}$

$$
\int_{S_{1}} v \phi d s=0 .
$$

Consider the following problem:

$$
\begin{aligned}
& \Delta \omega+k_{0}^{2} \omega=0, \quad \text { in } D_{0}, \\
& \Delta \omega+k_{1}^{2} \omega=0, \quad \text { in } D_{1}, \\
& \omega_{+}-\omega_{-}=0, \quad \text { on } S_{0} \text {, } \\
& \frac{\partial \omega_{+}}{\partial v}-\lambda_{0} \frac{\partial \omega_{-}}{\partial v}=0, \quad \text { on } S_{0} \\
& \frac{\partial \omega}{\partial \nu}+\operatorname{div}_{S_{1}}\left(\mu \nabla_{S_{1}} \omega\right)+\lambda \omega=\phi, \quad \text { on } S_{1}, \\
& \lim _{r \rightarrow \infty} \sqrt{r}\left(\frac{\partial \omega}{\partial r}-i k_{0} \omega\right)=0, \quad r=|x| .
\end{aligned}
$$

According to Theorem 7, this problem is well posedness and we have that the unique solution $\omega \in H_{\mathrm{loc}}^{1}\left(D_{0}\right) \cap H^{1}\left(D_{1}\right)$. Then we have

$$
\begin{aligned}
0 & =\int_{S_{1}} v \phi d s=\int_{S_{1}} v\left(\frac{\partial \omega}{\partial \nu}+\operatorname{div}_{S_{1}}\left(\mu \nabla_{S_{1}} \omega\right)+\lambda \omega\right) d s \\
& =\int_{S_{1}} v \frac{\partial \omega}{\partial \nu} d s+\int_{S_{1}} \omega\left(\operatorname{div}_{S_{1}}\left(\mu \nabla_{S_{1}} v\right)+\lambda v\right) d s \\
& =\int_{S_{1}}\left(v \frac{\partial \omega}{\partial v}-\omega \frac{\partial v}{\partial v}\right) d s .
\end{aligned}
$$

Furthermore, from the transmission boundary condition, Green's second theorem, and the radiation condition for $u^{s}$ and $\omega$ we have

$$
\begin{aligned}
0= & \frac{1}{\lambda_{0}} \int_{S_{0}}\left(u_{+} \frac{\partial \omega_{+}}{\partial \nu}-\omega_{+} \frac{\partial u_{+}}{\partial \nu}\right) d s \\
= & \frac{1}{\lambda_{0}} \int_{S_{0}}\left(u_{+}^{s} \frac{\partial \omega_{+}}{\partial \nu}-\omega_{+} \frac{\partial u_{+}^{s}}{\partial \nu}\right) d s \\
& +\frac{1}{\lambda_{0}} \int_{S_{0}}\left(u^{i} \frac{\partial \omega_{+}}{\partial \nu}-\omega_{+} \frac{\partial u^{i}}{\partial \nu}\right) d s \\
= & -\frac{1}{\gamma \lambda_{0}} \omega^{\infty}(-d) .
\end{aligned}
$$

Thus Rellich's lemma implies that $\omega=0$ in $D_{0}$. Then the transmission boundary conditions on $S_{0}$ and Holmgren's uniqueness theorem show that $\omega=0$ in $D_{1}$; hence $\phi=0$ from the trace theorem. We complete the proof of this lemma.

We are now in the position to present the uniqueness result based on the idea in [11]. 
Theorem 11. Given the interface $S_{0}$, the positive constant $\lambda_{0}$, and the wave numbers $k_{0}$ and $k_{1}$, due to incident plane waves $u^{i}=e^{i k_{0} x \cdot d}$, assume that the two far field patterns $u^{\infty}(\widehat{x}, d)$ and $\widetilde{u}^{\infty}(\hat{x}, d)$ corresponding to the two scattered fields $u^{s}(x, d)$ and $\widetilde{u}^{s}(x, d)$ which are arisen by two obstacles $D_{2}$ with impedances $(\mu, \lambda)$ and $\widetilde{D}_{2}$ with impedances $(\widetilde{\mu}, \widetilde{\lambda})$, respectively, coincide at a fixed frequency for all $\widehat{x} \in \mathbb{S}^{1}$ and $d \in \mathbb{S}^{1}$; then $D_{2}=\widetilde{D}_{2}$ and $(\mu, \lambda)=(\widetilde{\mu}, \tilde{\lambda})$.

Proof. If the obstacles are not the same, that is, $D_{2} \neq \widetilde{D}_{2}$, let $G$ be the unbounded component of $R^{2} \backslash\left(\bar{D}_{2} \cup \overline{\widetilde{D}}_{2}\right)$. By Rellich's lemma the scattered fields $u^{s}(\cdot, d)$ and $\widetilde{u}^{s}(x, d)$ corresponding to the incident plane wave $u^{i}(\cdot, d)$ coincide in the unbounded domain $G$. Without loss of generality, we may assume that there exist $z_{0}$ and open set $\Gamma$ such that $z_{0} \in \Gamma \subset \partial D_{2}$ and $\Gamma \cap \overline{\widetilde{D}}_{2}=\emptyset$. We can choose $h>0$ such that the sequence

$$
z_{j}=z_{0}+\frac{h}{j} \nu\left(z_{0}\right), \quad j=1,2, \ldots
$$

is contained in $G$, where $v\left(z_{0}\right)$ is the normal to $\partial D_{2}$ at $z_{0}$.

Consider the solution $u^{s}\left(\cdot, z_{j}\right)$ to problem (1) and (2) due to the incident point source $\Phi_{0}\left(\cdot, z_{j}\right)$. By Lemma 8, the far fields $\Phi_{0}^{\infty}\left(\cdot, z_{j}\right)$ and $\widetilde{\Phi}_{0}^{\infty}\left(\cdot, z_{j}\right)$ coincide in $G$. Then Rellich's lemma implies that $u^{s}\left(x, z_{j}\right)=\widetilde{u}^{s}\left(x, z_{j}\right)$ for $x \in G$. From this we have by denoting $P v=\partial v / \partial v+\operatorname{div}_{S_{1}}\left(\mu \nabla_{S_{1}} v\right)+\lambda v$

$$
P u^{s}\left(\cdot, z_{j}\right)=P \widetilde{u}^{s}\left(\cdot, z_{j}\right) \quad \text { on } \Gamma \text {. }
$$

Considering $\tilde{u}^{s}\left(x, z_{j}\right)$ as the scattered field corresponding to $\widetilde{D}_{2}$, using the boundary condition on $S_{1}$ for $u^{s}\left(x, z_{j}\right)$, we see that

$$
P \widetilde{u}^{s}\left(\cdot, z_{j}\right)=-P \Phi\left(\cdot, z_{j}\right) \quad \text { on } \Gamma \text {. }
$$

From the well posedness of problem (1) and (2) and the regularity of elliptic equations we obtain

$$
\begin{aligned}
\lim _{j \rightarrow \infty} P \widetilde{u}^{s}\left(\cdot, z_{j}\right)= & \frac{\partial \widetilde{u}^{s}\left(\cdot, z_{0}\right)}{\partial \nu}+\operatorname{div}_{S_{1}}\left(\mu \nabla_{S_{1}} \widetilde{u}^{s}\left(\cdot, z_{0}\right)\right) \\
& +\lambda \widetilde{u}^{s}\left(\cdot, z_{0}\right)
\end{aligned}
$$

in $L^{2}(\Gamma)$. On the other hand, as the same argument in Theorem 3.1 of [11], $P \Phi\left(\cdot, z_{0}\right)$ does not belong to $L^{2}(\Gamma)$. This is a contradiction, which implies that $D_{2}=\widetilde{D}_{2}$.

Next, we show that $(\mu, \lambda)=(\widetilde{\mu}, \tilde{\lambda})$. The proof is based on Theorem 3.1 in [11]; for the reader's convenience, we give its proof but make some slight modifications.

For this purpose, let $\widehat{\mu}=\mu-\widetilde{\mu}$ and $\widehat{\lambda}=\lambda-\widetilde{\lambda}$, and denote by $\widehat{v}$ the same total fields $v$ and $\widetilde{v}$. From the boundary conditions for the total field, we have that

$$
\operatorname{div}_{S_{1}}\left(\widehat{\mu} \nabla_{S_{1}} \widehat{v}\right)+\hat{\lambda} \widehat{v}=0 \quad \text { on } S_{1} .
$$

This equality should be understood in the weak sense. Thus for every $\theta \in H^{3 / 2}\left(S_{1}\right)$ we have

$$
\left\langle\widehat{v}, \operatorname{div}_{S_{1}}\left(\widehat{\mu} \nabla_{S_{1}} \theta\right)+\hat{\lambda} \theta\right\rangle_{H^{3 / 2}\left(S_{1}\right), H^{-3 / 2}\left(S_{1}\right)}=0 .
$$

With the help of Lemma 10, we obtain

$$
\operatorname{div}_{S_{1}}\left(\widehat{\mu} \nabla_{S_{1}} \theta\right)+\hat{\lambda} \theta=0, \quad \forall \theta \in H^{3 / 2}\left(S_{1}\right) .
$$

Choosing $\theta=1$ in the above equation leads to $\hat{\lambda}=0$. The above equation also implies that

$$
\int_{S_{1}} \widehat{\mu}\left|\nabla_{S_{1}} \theta\right|^{2} d s=0, \quad \forall \theta \in H^{3 / 2}\left(S_{1}\right) .
$$

Assume that $\widehat{\mu}\left(x_{0}\right) \neq 0$ for some $x_{0} \in S_{1}$; then, for example, $\operatorname{Re} \widehat{\mu}\left(x_{0}\right)>0$ without loss of generality. Since $\widehat{\mu}$ is continuous there exists $\varepsilon>0$ such that $\operatorname{Re} \widehat{\mu}(x)>0$ for all $x \in$ $S_{1} \cap B\left(x_{0} ; \varepsilon\right)$. Let us choose $\theta$ as a smooth and compactly supported function in $S_{1} \cap B\left(x_{0} ; \varepsilon\right)$; we obtain that

$$
\int_{S_{1} \cap B\left(x_{0} ; \varepsilon\right)} \operatorname{Re}(\widehat{\mu})\left|\nabla_{S_{1}} \theta\right|^{2} d s=0,
$$

and then $\nabla_{S_{1}} \theta=0$ on $S_{1} \cap B\left(x_{0} ; \varepsilon\right)$; that is, $\theta$ is a constant on $S_{1} \cap B\left(x_{0} ; \varepsilon\right)$, which is a contradiction. We hence have $\widehat{\mu}=0$ on $S_{1}$ and the proof is completed.

\section{The Modified Linear Sampling Method}

In this part, we give a mathematical basis to reconstruct the shape of the obstacle $D_{2}$ by using the modified linear sampling method (see [23]).

We do some preparation firstly. Consider the total wave $u_{0}=u^{i}+u_{0}^{s}\left(u^{i}(x, d)=e^{i k_{0} x \cdot d}\right)$ such that

$$
\begin{aligned}
& \Delta u_{0}+k_{0}^{2} u_{0}=0, \quad \text { in } D_{0}, \\
& \Delta u_{0}+k_{1}^{2} u_{0}=0, \quad \text { in } D, \\
& u_{0+}-u_{0-}=0, \quad \text { on } S_{0} \text {, } \\
& \frac{\partial u_{0+}}{\partial \nu}-\lambda_{0} \frac{\partial u_{0-}}{\partial \nu}=0, \quad \text { on } S_{0}, \\
& \lim _{r \rightarrow \infty} \sqrt{r}\left(\frac{\partial u_{0}^{s}}{\partial r}-i k_{0} u_{0}^{s}\right)=0, \quad r=|x| .
\end{aligned}
$$

Recalling that $u(x, d)$ and $v(x, d)$ are the solution to scattering problem (1) and (2) for incident plane wave $u^{i}(x, d)=e^{i k_{0} x \cdot d}$ with the direction $d \in \mathbb{S}^{1}$, it is easy to verify that the fields $w(x):=u-u_{0}, x \in D_{0}$ and $z(x):=v-u_{0}, x \in D_{1}$ solve the following boundary value problem:

$$
\begin{aligned}
\Delta w+k_{0}^{2} w=0, & \text { in } D_{0}, \\
\Delta z+k_{1}^{2} z=0, & \text { in } D_{1}, \\
w_{+}-z_{-}=0, & \text { on } S_{0}, \\
\frac{\partial w_{+}}{\partial v}-\lambda_{0} \frac{\partial z_{-}}{\partial v}=0, & \text { on } S_{0}, \\
\frac{\partial z}{\partial \nu}+\operatorname{div}_{S_{1}}\left(\mu \nabla_{S_{1}} z\right)+\lambda z=\phi, & \text { on } S_{1}, \\
\lim _{r \rightarrow \infty} \sqrt{r}\left(\frac{\partial w}{\partial r}-i k_{0} w\right)=0, & r=|x|,
\end{aligned}
$$


where $\phi=-\left(\partial u_{0} / \partial \nu\right)-\operatorname{div}_{S_{1}}\left(\mu \nabla_{S_{1}} u_{0}\right)-\lambda u_{0}$. The well posedness of this boundary value problem has been established in Section 1.

We define four operators in the following.

The data-to-pattern operator $G: H^{-3 / 2}\left(S_{1}\right) \rightarrow L^{2}\left(\mathbb{S}^{1}\right)$ by

$$
G(\phi)(\widehat{x})=w^{\infty},
$$

where $w^{\infty}$ is the far field pattern of the wave field $w$ of problem (64).

The auxiliary operator $H: L^{2}\left(\mathbb{S}^{1}\right) \rightarrow H^{-3 / 2}\left(S_{1}\right)$ by

$$
\begin{aligned}
& (H g)(x) \\
& \quad=\int_{\mathbb{S}^{1}}\left(\frac{\partial u_{0}(x, d)}{\partial \nu(x)}+\operatorname{div}_{S_{1}}\left(\mu \nabla_{S_{1}} u_{0}\right)+i \lambda u_{0}(x, d)\right) \\
& \quad \cdot g(d) d s(d)
\end{aligned}
$$

where $u_{0}$ is the solution of (63) corresponding to the incident wave $u^{i}(\cdot, d)$.

The far field operator $F: L^{2}\left(\mathbb{S}^{1}\right) \rightarrow L^{2}\left(\mathbb{S}^{1}\right)$ by

$$
(F g)(\widehat{x})=\int_{\mathbb{S}^{1}} u^{\infty}(\widehat{x}, d) g(d) d s(d),
$$

where $u^{\infty}$ is the far field pattern of the scattered wave $u^{s}$ of problem (1) and (2).

The far field operator $F_{0}: L^{2}\left(\mathbb{S}^{1}\right) \rightarrow L^{2}\left(\mathbb{S}^{1}\right)$ by

$$
\left(F_{0} g\right)(\widehat{x})=\int_{\mathbb{S}^{1}} u_{0}^{\infty}(\widehat{x}, d) g(d) d s(d),
$$

where $u_{0}^{\infty}$ is the far field pattern of the scattered wave $u_{0}^{s}$ of problem (63).

Note that

$$
\begin{aligned}
& {\left[\left(F-F_{0}\right) g\right](\widehat{x})} \\
& \quad=\int_{\mathbb{S}^{1}}\left[u^{\infty}(\widehat{x}, d)-u_{0}^{\infty}(\widehat{x}, d)\right] g(d) d s(d)
\end{aligned}
$$

is just the far field pattern of the radiating function

$$
\begin{aligned}
& \int_{\mathbb{S}^{1}}\left\{\left[u(x, d)-u^{i}(x, d)\right]-\left[u_{0}(x, d)-u^{i}(x, d)\right]\right\} \\
& \cdot g(d) d s(d)=\int_{\mathbb{S}^{1}}\left[u(x, d)-u_{0}(x, d)\right] \\
& \cdot g(d) d s(d) .
\end{aligned}
$$

From the boundary conditions on $S_{1}$ for $v(x, d)$ and $z(x, d)$, we can factorize the operator $F-F_{0}$ as

$$
F-F_{0}=-G H \text {. }
$$

Let $G_{0}(\cdot, m), m \in R^{2}$ be the Green function for problem (63) of scattering by the background medium. We now define the modified far field equation

$$
\begin{aligned}
& \left(F-F_{0}\right)\left(g_{m}\right)(\widehat{x})=G_{0}^{\infty}(\widehat{x}, m) \\
& \qquad \text { for } g \in L^{2}\left(\mathbb{S}^{1}\right), \hat{x} \in \mathbb{S}^{1},
\end{aligned}
$$

where $G_{0}^{\infty}(\widehat{x}, m) \in L^{2}\left(\mathbb{S}^{1}\right)$ is the far field pattern of the Green function $G_{0}(x, m)$. We will characterize the obstacle $D_{2}$ by the behavior of an approximate solution $g_{m}$ of far field equation (72).

To prove the existence of an approximate solution of (72), we firstly explore the related properties of the operators $G$ and $H$.

Lemma 12. The data-to-pattern operator $G: H^{-3 / 2}\left(S_{1}\right) \rightarrow$ $L^{2}\left(\mathbb{S}^{1}\right)$ is injective and compact and has dense range in $L^{2}\left(\mathbb{S}^{1}\right)$.

Proof. First, injectivity is a direct consequence of Rellich's lemma and analytic continuation of the solution to (64).

To prove compactness, using Green's representation formula for $z$ in $D_{1}$ and $w$ in $D_{0}$, we can decompose the operator $G$ as $G=G_{1} G_{2}$, where $G_{2}: H^{-3 / 2}\left(S_{1}\right) \rightarrow H^{1 / 2}\left(S_{0}\right) \times$ $H^{-1 / 2}\left(S_{0}\right)$ is defined by $G_{2}(\phi)=\left(\left.z_{-}\right|_{S_{0}},\left.\lambda_{0}\left(\partial z_{-} / \partial \nu\right)\right|_{S_{0}}\right)$ and $G_{1}: H^{1 / 2}\left(S_{0}\right) \times H^{-1 / 2}\left(S_{0}\right) \rightarrow L^{2}\left(\mathbb{S}^{1}\right)$ is defined by

$$
\begin{aligned}
& G_{1}\left(w_{+}, \frac{\partial w_{+}}{\partial \nu}\right)(\widehat{x}) \\
& =\gamma \int_{S_{0}}\left(w_{+}(y) \frac{\partial e^{-i k_{0} \hat{x} \cdot y}}{\partial \nu(y)}-\frac{\partial w_{+}(y)}{\partial \nu(y)} e^{-i k_{0} \hat{x} \cdot y}\right) d s(y),
\end{aligned}
$$

$$
\widehat{x} \in \mathbb{S}^{1},
$$

where $\gamma=e^{i \pi / 4} / \sqrt{8 k_{0} \pi}$. The interior regularity of the solution to problem (64) implies that the operator $G_{2}$ is bounded. So the operator $G$ is compact since the operator $G_{1}$ is compact.

To show denseness of the range of $G$ we just need to prove that the adjoint operator $G^{*}$ is injective. To this end, let $U$ and $V$ be the solution of problem (1) and (2) with incident plane wave

$$
U^{i}=\int_{\mathbb{S}^{1}} e^{-i k_{0} \hat{x} \cdot y} \overline{g(\widehat{x})} d s(\widehat{x}), \quad g \in L^{2}\left(\mathbb{S}^{1}\right) .
$$

We remind the reader that $U \in H_{\mathrm{loc}}^{2}\left(D_{0}\right)$ and $V \in H^{2}\left(D_{1}\right)$ by the regularity of elliptic equations.

For any $\phi \in H^{-3 / 2}\left(S_{1}\right)$, let $w$ and $z$ be the solution to problem (64) with the boundary data $\phi$. Then one can derive that by Green's second theorem and the radiation condition of scattered field

$$
\begin{aligned}
\left\langle\phi, G^{*} g\right\rangle_{H^{-3 / 2}\left(S_{1}\right), H^{3 / 2}\left(S_{1}\right)}=\langle G \phi, g\rangle_{L^{2}\left(\mathbb{S}^{1}\right), L^{2}\left(\mathbb{S}^{1}\right)} & \int_{\mathbb{S}^{1}} w^{\infty}(\widehat{x}) \overline{g(\widehat{x})} d s(\widehat{x}) \\
& =\gamma \int_{\mathbb{S}^{1}} \int_{S_{0}}\left(w_{+}(y) \frac{\partial e^{-i k_{0} \hat{x} \cdot y}}{\partial \nu(y)}\right. \\
& -\frac{\partial w_{+}(y)}{\partial \nu(y)} e^{\left.-i k_{0} \hat{x} \cdot y\right) d s(y) \overline{g(\widehat{x})} d s(\widehat{x})} \\
& =\gamma \int_{S_{0}}\left(w_{+}(y) \frac{\partial U^{i}(y)}{\partial \nu(y)}-\frac{\partial w_{+}(y)}{\partial \nu(y)} U^{i}(y)\right) d s(y) \\
& =\gamma \int_{S_{0}}\left(w_{+}(y) \frac{\partial U_{+}(y)}{\partial \nu(y)}-\frac{\partial w_{+}(y)}{\partial \nu(y)}\right.
\end{aligned}
$$




$$
\begin{aligned}
& \left.\cdot U_{+}(y)\right) d s(y)=\gamma \lambda_{0} \int_{S_{1}}\left(z(y) \frac{\partial V(y)}{\partial \nu(y)}\right. \\
& \left.-\frac{\partial z(y)}{\partial \nu(y)} V(y)\right) d s(y)=-\gamma \lambda_{0} \int_{S_{1}}(z(y) \\
& \cdot\left(\operatorname{div}_{S_{1}}\left(\mu \nabla_{S_{1}} V(y)\right)+\lambda V(y)\right)+\frac{\partial z(y)}{\partial \nu(y)} \\
& \cdot V(y)) d s(y)=-\gamma \lambda_{0} \int_{S_{1}}\left(\frac{\partial z(y)}{\partial \nu(y)}\right. \\
& \left.+\operatorname{div}_{S_{1}}\left(\mu \nabla_{S_{1}} z(y)\right)+\lambda z(y)\right) V(y) d s(y) \\
& =-\gamma \lambda_{0} \int_{S_{1}} \phi(y) V(y) d s(y) .
\end{aligned}
$$

Thus we obtain that

$$
G^{*} g=-\left.\lambda_{0} \bar{\gamma} \bar{V}\right|_{S_{1}} .
$$

Letting $G^{*} g=0$, we have $\left.V\right|_{S_{1}}=0$ and then $\left.(\partial V / \partial \nu)\right|_{S_{1}}=$ 0 by the GIBC. Holmgren's uniqueness theorem implies that $V=0$ in $D_{1}$ and then $U=0$ in $D_{0}$; this leads to $U^{i}=0$ since $U^{i}$ does not satisfy the radiation condition; however $U^{s}$ does. Then we can conclude that $g=0$ which means that $G^{*}$ is injective. We complete the proof of this theorem.

Lemma 13. Let $G$ be defined by (65), for any $m \in R^{2}$; then $G_{0}^{\infty}(\widehat{x}, m) \in L^{2}\left(\mathbb{S}^{1}\right)$ belongs to the range of $R\left(G_{0}\right)$ if and only if $m \in D_{2}$.

Proof. Firstly, let $m \in D_{2}$; then $G_{0}(x, m)$ satisfies problem (64) with

$$
\phi=\left.\left(\frac{\partial G_{0}}{\partial \nu}+\operatorname{div}_{S_{1}}\left(\mu \nabla_{S_{1}} G_{0}\right)+\lambda G_{0}\right)\right|_{S_{1}} .
$$

By the definition of the operator $G$, we get $G \phi$ coinciding with $G_{0}^{\infty}$.

Now let $m \notin D_{2}$ and assume on the contrary that there exists $\phi \in H^{-3 / 2}\left(S_{1}\right)$ such that $G \phi=G_{0}^{\infty}$. Let $w, z$ be the solution of problem (64) with boundary data $\phi$; then $w^{\infty}=G_{0}^{\infty}$. By Rellich's lemma we conclude that $w(x)=$ $G_{0}(x, z)$ in $D_{0} \backslash\{m\}$ and then as a direct derivation $z=$ $G_{0}(x, z)$ in $D_{1} \backslash\{m\}$.

If $m \in D_{1}$, this contradicts the fact that $z$ is analytic in $D_{0}$ but $G_{0}(x, m)$ is singular at $x=m$. If $m \in S_{1}$, by the well posedness of the direct problem $\|z\|_{H^{1 / 2}\left(S_{1}\right)}$ is bounded which contradicts that $\left\|G_{0}\right\|_{H^{1 / 2}\left(S_{1}\right)}=\infty$. For the case $m \in D_{0}$ or $m \in S_{0}$ we also can infer a contradiction, so we complete the proof of this lemma.

Now, we turn our attention to the operator $H$. In order to obtain the required properties of the operator $H$, we need the following two results in [23].

Lemma 14 (mixed reciprocity relation). For $\widehat{x} \in \mathbb{S}^{1}$, we have

$$
G_{0}^{\infty}(\widehat{x}, m)= \begin{cases}u_{0}(m,-\widehat{x}), & m \in D_{0}, \hat{x} \in \mathbb{S}^{1}, \\ \lambda_{0} u_{0}(m,-\widehat{x}), & m \in D, \hat{x} \in \mathbb{S}^{1}\end{cases}
$$

where $G_{0}^{\infty}(\hat{x}, m)$ is the far field pattern of Green function $G_{0}(x, m)$ and $u_{0}(m,-\widehat{x})$ is the solution of (63) with incident plane wave $u^{i}(m,-\widehat{x})$.

Lemma 15. For all $m \in D, \widehat{x} \in \mathbb{S}^{1}$, we have

$$
u_{0}(m,-\widehat{x})=\left(\mathcal{S}_{0} \overline{u_{0}(m, \cdot)}\right)(\widehat{x})
$$

where $\mathcal{S}_{0}=I+2 i k_{0}|\gamma|^{2} F_{0}$ introduced in [23] is the scattering operator.

Lemma 16. If $\operatorname{Im}(\lambda) \neq \equiv$ on $S_{1}$, then the operator $H$ defined by (66) has dense range.

Proof. The adjoint operator of $H$ is $H^{*}: H^{3 / 2}\left(S_{1}\right) \rightarrow L^{2}\left(\mathbb{S}^{1}\right)$ with

$$
\begin{aligned}
& H^{*}(\xi)(\widehat{x})=\int_{S_{1}}\left(\overline{\frac{\partial u_{0}(y, \widehat{x})}{\partial \nu(y)}}+\operatorname{div}_{S_{1}}\left(\bar{\mu} \nabla_{S_{1}} \overline{u_{0}(y, \widehat{x})}\right)\right. \\
& \left.\quad+\bar{\lambda} \overline{u_{0}(y, \widehat{x})}\right) \xi(y) d s(y) .
\end{aligned}
$$

We just need to show that the operator $H^{*}$ is injective. Using Lemmas 14 and 15, we have for every $\xi \in H^{3 / 2}\left(S_{1}\right)$

$$
\begin{aligned}
\left(\lambda_{0} \mathcal{S}_{0} H^{*}(\xi)\right)(\widehat{x}) & =\lambda_{0}\left\{\mathcal{S}_{0}\left(\int_{S_{1}}\left(\frac{\overline{\partial u_{0}(y, \cdot)}}{\partial \nu(y)}+\operatorname{div}_{S_{1}}\left(\bar{\mu} \nabla_{S_{1}} \overline{u_{0}(y, \cdot)}\right)+\bar{\lambda} \overline{u_{0}(y, \cdot)}\right) \xi(y) d s(y)\right)\right\} \\
& =\lambda_{0}\left\{\int_{S_{1}}\left(\frac{\partial u_{0}(y,-\widehat{x})}{\partial \nu(y)}+\operatorname{div}_{S_{1}}\left(\bar{\mu} \nabla_{S_{1}} u_{0}(y,-\widehat{x})\right)+\bar{\lambda} u_{0}(y,-\widehat{x})\right) \xi(y) d s(y)\right\} \\
& =\int_{S_{1}}\left(\frac{\partial G_{0}^{\infty}(\widehat{x}, y)}{\partial \nu(y)}+\operatorname{div}_{S_{1}}\left(\bar{\mu} \nabla_{S_{1}} G_{0}^{\infty}(\widehat{x}, y)\right)+\bar{\lambda} G_{0}^{\infty}(\widehat{x}, y)\right) \xi(y) d s(y) .
\end{aligned}
$$


Therefore, we conclude that $\lambda_{0} \mathcal{S}_{0} H^{*}(\xi)$ is just the far field pattern of the potential

$$
\begin{aligned}
& W(x)=\int_{S_{1}}\left(\frac{\partial G_{0}(x, y)}{\partial \nu(y)}+\operatorname{div}_{S_{1}}\left(\bar{\mu} \nabla_{S_{1}} G_{0}(x, y)\right)\right. \\
& \left.+\bar{\lambda} G_{0}(x, y)\right) \xi(y) d s(y) \\
& =\int_{S_{1}} \frac{\partial G_{0}(x, y)}{\partial \nu(y)} \xi(y)+G_{0}(x, y)(\bar{\lambda} \xi(y) \\
& \left.+\operatorname{div}_{S_{1}}\left(\bar{\mu} \nabla_{S_{1}} \xi(y)\right)\right) d s(y)
\end{aligned}
$$

for $x \in R^{2} \backslash S_{1}$.

Due to the fact that $\mathcal{S}_{0}$ is unitary (see [24]), we just need to show that $\xi=0$ under the assumption $W^{\infty}(\widehat{x})=0$. Next we will prove this assertion.

In fact, if $W^{\infty}(\widehat{x})=0$, then Rellich's lemma implies that $W(x)=0$ for $x \in R^{2} \backslash \bar{D}_{2}$. Then by using the jump relations of single- and double-potentials, we get

$$
\begin{aligned}
\left.W_{-}\right|_{S_{1}} & =\left.W_{-}\right|_{S_{1}}-\left.W_{+}\right|_{S_{1}}=-\xi, \\
\left.\frac{\partial W_{-}}{\partial \nu}\right|_{S_{1}} & =\left.\frac{\partial W_{-}}{\partial \nu}\right|_{S_{1}}-\left.\frac{\partial W_{+}}{\partial \nu}\right|_{S_{1}}=\bar{\lambda} \xi+\operatorname{div}_{S_{1}}\left(\bar{\mu} \nabla_{S_{1}} \xi\right) .
\end{aligned}
$$

Thus $\bar{W}$ satisfies the Helmholtz equation in $D_{2}$ with GIBC:

$$
\begin{aligned}
\Delta \bar{W}+k_{1}^{2} \bar{W}=0, & \text { in } D_{2}, \\
\frac{\partial \bar{W}}{\partial \nu}+\operatorname{div}_{S_{1}}\left(\mu \nabla_{S_{1}} \bar{W}\right)+\lambda \bar{W}=0, & \text { on } S_{1} .
\end{aligned}
$$

By the assumption on $\lambda$, there exists $x_{0} \in S_{1}$ such that $\operatorname{Im}(\lambda(x)) \neq 0$ in a small neighborhood $\Lambda\left(x_{0}\right) \subset S_{0}$. Green's theorem in $D_{2}$ and the divergence theorem on $S_{1}$ imply

$$
\begin{aligned}
0= & \int_{D_{2}}\left(\Delta W+k_{1}^{2} W\right) \bar{W} d x \\
= & -\int_{D_{2}}\left(|\nabla W|^{2}-k_{1}^{2}|W|^{2}\right) d x+\int_{S_{1}} \bar{\mu}\left|\frac{d W}{d s}\right|^{2} d s \\
& -\int_{S_{1}} \bar{\lambda}|W|^{2} d s .
\end{aligned}
$$

By taking the imaginary part of the above equation we can obtain that $W=0$ on $\Lambda\left(x_{0}\right)$; then the boundary condition shows that $\partial W / \partial \nu=0$ on $\Lambda\left(x_{0}\right)$. Thus Holmgren's uniqueness theorem implies that $W=0$ in $D_{2}$; we then obtain $\xi=0$ from the jump relations. This lemma is then proved.

Finally, we give the main result in this paper, that is, recovering the obstacles $D_{2}$ by a modified linear sampling method.

Theorem 17. Under Assumption A, then we have the following results:
(1) If $m \in D_{2}$, then for every $\epsilon>0$ there exists a solution $g_{m}^{\epsilon} \in L^{2}\left(\mathbb{S}^{1}\right)$ to the far field equation (72) such that

$$
\left\|\left(F-F_{0}\right) g_{m}^{\epsilon}+G_{0}^{\infty}\right\|_{L^{2}\left(\mathbb{S}^{1}\right)}<\epsilon .
$$

(2) If $m \notin D_{2}$, then for every $\epsilon>0$ and $\delta>0$ there exists a function $g_{m}^{\epsilon, \delta} \in L^{2}\left(\mathbb{S}^{1}\right)$ such that

$$
\begin{array}{r}
\left\|\left(F-F_{0}\right) g_{m}^{\epsilon, \delta}+G_{0}^{\infty}\right\|_{L^{2}\left(\mathbb{S}^{1}\right)}<\epsilon+\delta, \\
\lim _{\delta \rightarrow 0}\left\|g_{m}^{\epsilon, \delta}\right\|_{L^{2}\left(\mathbb{S}^{1}\right)}=\infty .
\end{array}
$$

Proof. If $m \in D_{2}$, by using Lemma 13 there exists $\phi \epsilon$ $H^{-3 / 2}\left(S_{1}\right)$ such that $G \phi=G_{0}^{\infty}$. From Lemma 16, for every $\epsilon_{0}>0$ there exists a function $g_{m}^{\epsilon_{0}} \in L^{2}\left(\mathbb{S}^{1}\right)$ such that

$$
\left\|H g_{m}^{\epsilon_{0}}-\phi\right\|_{H^{-1 / 2}\left(S_{1}\right)}<\epsilon_{0} .
$$

The operator $G$ is bounded from Lemma 12; then we have

$$
\left\|G H g_{m}^{\epsilon_{0}}-G \phi\right\|_{L^{2}\left(\mathbb{S}^{1}\right)}<c_{1} \epsilon_{0},
$$

where $c_{1}$ is a constant; that is,

$$
\left\|\left(F-F_{0}\right) g_{m}^{\epsilon}+G_{0}^{\infty}\right\|_{L^{2}\left(\mathbb{S}^{1}\right)}<\epsilon,
$$

where $\epsilon=c_{1} \epsilon_{0}$.

Next, we assume that $m \notin D_{2}$. In this case, by Lemma $13 G_{0}^{\infty}$ is not in the range of $G$. But from Lemma 12 we know that the operator $G$ is compact and injective with dense range in $L^{2}\left(\mathbb{S}^{1}\right)$. Hence for every $\delta>0$ we can construct a unique Tikhonov regularized solution $\phi^{\rho} \in H^{-3 / 2}\left(S_{1}\right)$ of equation $G \phi^{\rho}=G_{0}^{\infty}$, such that

$$
\left\|G \phi^{\rho}-G_{0}^{\infty}\right\|_{L^{2}\left(\mathbb{S}^{1}\right)}<\delta,
$$

where $\rho$ is the regularization parameter (chosen by a regular regularization strategy, e.g., the Morozov discrepancy principle [25]). Then we have $\left\|\phi^{\rho}\right\|_{H^{-3 / 2}\left(S_{1}\right)} \rightarrow \infty$ as $\rho \rightarrow 0$. By Lemma $16 H$ has dense range; hence for $\epsilon>0$ sufficiently small there exists $g_{m}^{\epsilon, \rho}$ such that

$$
\left\|H g_{m}^{\epsilon, \rho}-\phi^{\rho}\right\|_{H^{-3 / 2}\left(S_{1}\right)}<\frac{\epsilon}{c_{1}} .
$$

Combining (91) and (92) we obtain that for every $\epsilon>0$ and $\delta>0$ there exists $g_{m}^{\epsilon, \rho} \in L^{2}\left(\mathbb{S}^{1}\right)$ such that

$$
\begin{aligned}
& \left\|\left(F-F_{0}\right) g_{m}^{\epsilon, \rho}+G_{0}^{\infty}\right\|_{L^{2}\left(\mathbb{S}^{1}\right)}=\left\|G H g_{m}^{\epsilon, \rho}-G_{0}^{\infty}\right\|_{L^{2}\left(\mathbb{S}^{1}\right)} \\
& \quad \leq\left\|G H g_{m}^{\epsilon, \rho}-G \phi^{\rho}\right\|_{L^{2}\left(\mathbb{S}^{1}\right)}+\left\|G \phi^{\rho}-G_{0}^{\infty}\right\|_{L^{2}\left(\mathbb{S}^{1}\right)} \\
& \quad<\epsilon+\delta .
\end{aligned}
$$

Since $\lim _{\delta \rightarrow 0} \rho(\delta)=0$ we have that $\lim _{\delta \rightarrow 0}\left\|\phi^{\rho}\right\|_{H^{-3 / 2}\left(S_{1}\right)} \rightarrow$ $\infty$. From (92) we have that $\lim _{\delta \rightarrow 0}\left\|H g_{m}^{\epsilon, \delta}\right\|_{H^{-3 / 2}\left(S_{1}\right)} \rightarrow \infty$. By the definition of the operator $H$ given by (66) we obtain that $\lim _{\delta \rightarrow 0}\left\|g_{m}^{\epsilon, \delta}\right\|_{L^{2}\left(\mathbb{S}^{1}\right)} \rightarrow \infty$. Then we complete the proof of this theorem. 
Remark 18. (1) From Theorem 17, we have to obtain the far field pattern of Green function $G_{0}(x, m)$ which is defined in the layered background medium. Typically, this is a quite difficult task; however, with the help of Lemma 14 we only need to solve transmission problem (63) to get $u_{0}$ instead of $G_{0}$.

(2) In this paper, we just consider the case of two-layered background medium; in fact, our result can be extended to the case of multilayered piecewise homogeneous medium.

\section{Conflict of Interests}

The authors declare that there is no conflict of interests regarding the publication of this paper.

\section{Acknowledgments}

This research is supported by NSFC Grant no. 11171127 and NSFC Grant no. 11571132. This research is also supported by the Fundamental Research Funds for the Central Universities, nos. CZQ15020 and CZQ12014.

\section{References}

[1] A. Henrot and M. Pierre, Variation et Optimisation de Formes: Une Analyse Géométrique, vol. 48 of Mathématiques et Applications, Springer, Berlin, Germany, 2005.

[2] D. Colton and R. Kress, Inverse Acoustic and Electromagnetic Scattering Theory, Springer, Berlin, Germany, 2nd edition, 1998.

[3] L. Bourgeois, N. Chaulet, and H. Haddar, "Identication of generalized impedance boundary conditions: some numerical issues," Tech. Rep. 7449, INRIA, 2010.

[4] J. Yang, B. Zhang, and H. Zhang, "Reconstruction of complex obstacles with generalized impedance boundary conditions from far-field data," SIAM Journal on Applied Mathematics, vol. 74, no. 1, pp. 106-124, 2014.

[5] D. S. Jones, "Integral equations for the exterior acoustic problem," The Quarterly Journal of Mechanics and Applied Mathematics, vol. 27, pp. 129-142, 1974.

[6] X. Liu and B. Zhang, "Direct and inverse obstacle scattering problems in a piecewise homogeneous medium," SIAM Journal on Applied Mathematics, vol. 70, no. 8, pp. 3105-3120, 2010.

[7] X. Liu, B. Zhang, and G. Hu, "Uniqueness in the inverse scattering problem in a piecewise homogeneous medium," Inverse Problems, vol. 26, no. 1, Article ID 015002, 2010.

[8] A. Kirsch and L. Päivärinta, "On recovering obstacles inside inhomogeneities," Mathematical Methods in the Applied Sciences, vol. 21, no. 7, pp. 619-651, 1998.

[9] P. Hahner, "A uniqueness theorem for an inverse scattering problem in an exterior domain," SIAM Journal on Mathematical Analysis, vol. 29, no. 5, pp. 1118-1128, 1998.

[10] A. I. Nachman, L. Päivärinta, and A. Teirilä, "On imaging obstacles inside inhomogeneous media," Journal of Functional Analysis, vol. 252, no. 2, pp. 490-516, 2007.

[11] L. Bourgeois, N. Chaulet, and H. Haddar, "On simultaneous identification of the shape and generalized impedance boundary condition in obstacle scattering," SIAM Journal on Scientific Computing, vol. 34, no. 3, pp. A1824-A1848, 2012.
[12] D. Colton and A. Kirsch, "A simple method for solving inverse scattering problems in the resonance region," Inverse Problems, vol. 12, no. 4, pp. 383-393, 1996.

[13] D. Colton, H. Haddar, and M. Piana, "The linear sampling method in inverse electromagnetic scattering theory," Inverse Problems, vol. 19, no. 6, pp. S105-S137, 2003.

[14] D. Colton, J. Coyle, and P. Monk, "Recent developments in inverse acoustic scattering theory," SIAM Review, vol. 42, no. 3, pp. 369-414, 2000.

[15] F. Cakoni, M. di Cristo, and J. Sun, "A multistep reciprocity gap functional method for the inverse problem in a multilayered medium," Complex Variables and Elliptic Equations, vol. 57, no. 2-4, pp. 261-276, 2012.

[16] D. Colton and H. Haddar, "An application of the reciprocity gap functional to inverse scattering theory," Inverse Problems, vol. 21, no. 1, pp. 383-398, 2005.

[17] H. Zhang and B. Zhang, "A Newton method for a simultaneous reconstruction of an interface and a buried obstacle from farfield data," Inverse Problems, vol. 29, no. 4, 2013.

[18] R. Kress, Acoustic Scattering: Special Theoretical Tools Scattering, edited by: R. Pike, P. Sabatier, Academic Press, London, UK, 2001.

[19] D. Colton and R. Kress, Integral Equation Methods in Scattering Theory, Springer, Wiley, New York, NY, USA, 1983.

[20] W. McLean, Strongly Elliptic Systems and Boundary Integral Equation, Cambridge University Press, Cambridge, UK, 2000.

[21] F. Cakoni and R. Kress, "Integral equation methods for the inverse obstacle problem with generalized impedance boundary condition," Inverse Problems, vol. 29, no. 1, Article ID 015005, 19 pages, 2013.

[22] L. C. Evans, Partial Differential Equations, American Mathematical Society, Providence, RI, USA, 1998.

[23] O. Bondarenko, A. Kirsch, and X. Liu, "The factorization method for inverse acoustic scattering in a layered medium," Inverse Problems, vol. 29, no. 4, Article ID 045010, 19 pages, 2013.

[24] A. Kirsch and N. Grinberg, The Factorization Method for Inverse Problems, vol. 36 of Oxford Lecture Series in Mathematics and its Applications, Oxford University Press, Oxford, UK, 2008.

[25] D. Colton, M. Piana, and R. Potthast, "A simple method using Morozov's discrepancy principle for solving inverse scattering problems," Inverse Problems, vol. 13, no. 6, pp. 1477-1493, 1997. 


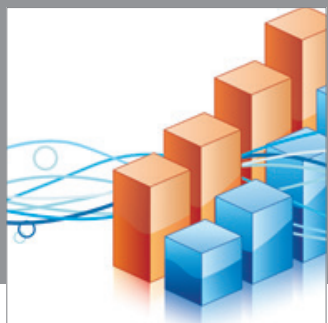

Advances in

Operations Research

mansans

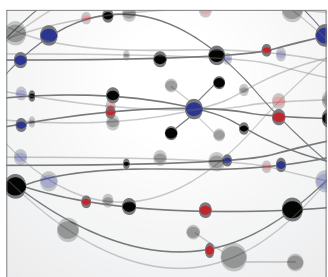

The Scientific World Journal
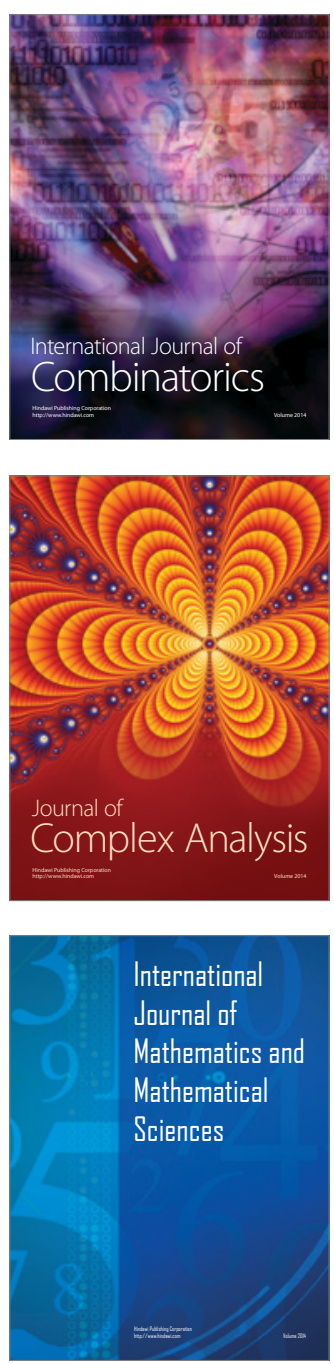
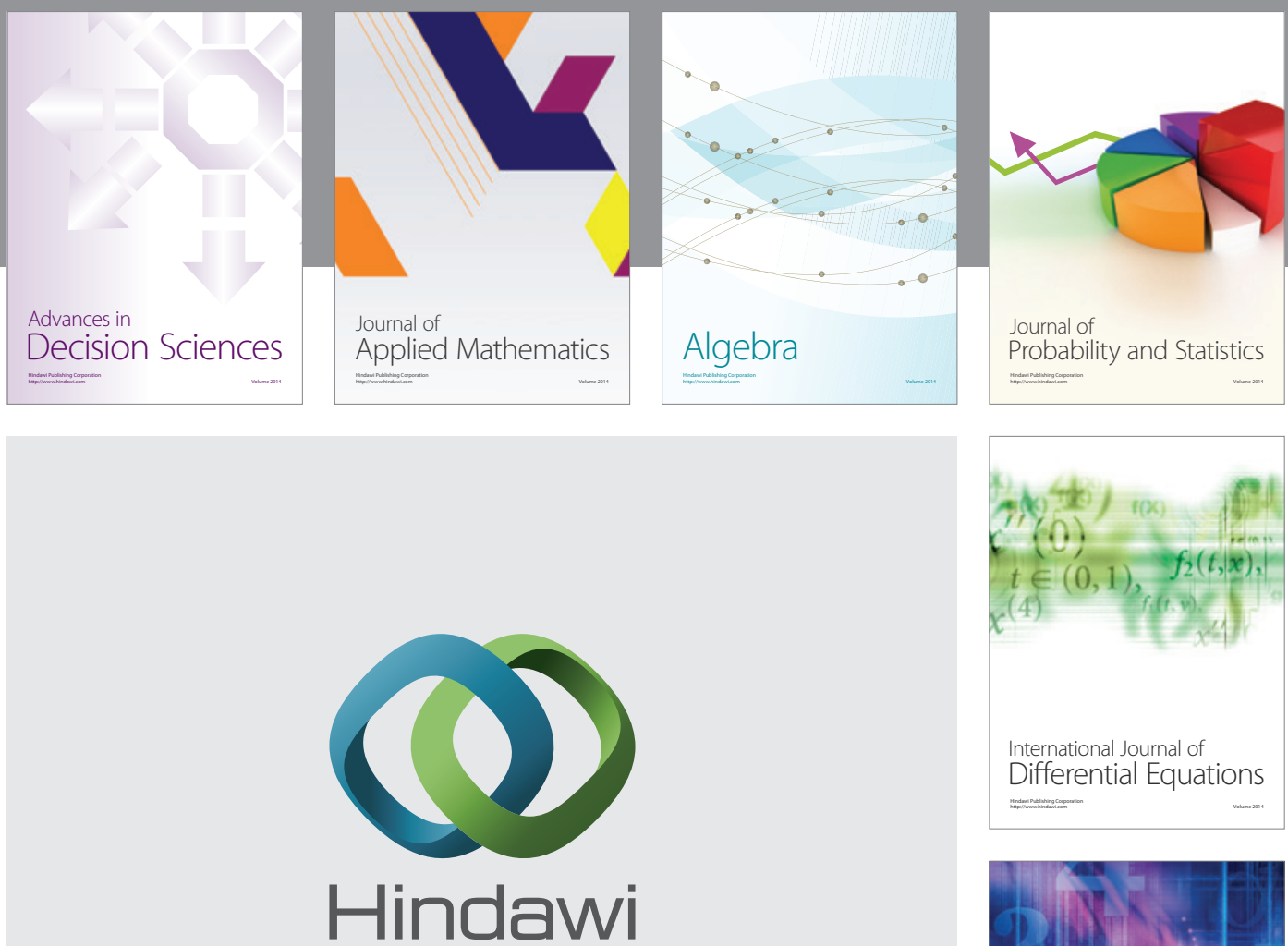

Submit your manuscripts at http://www.hindawi.com
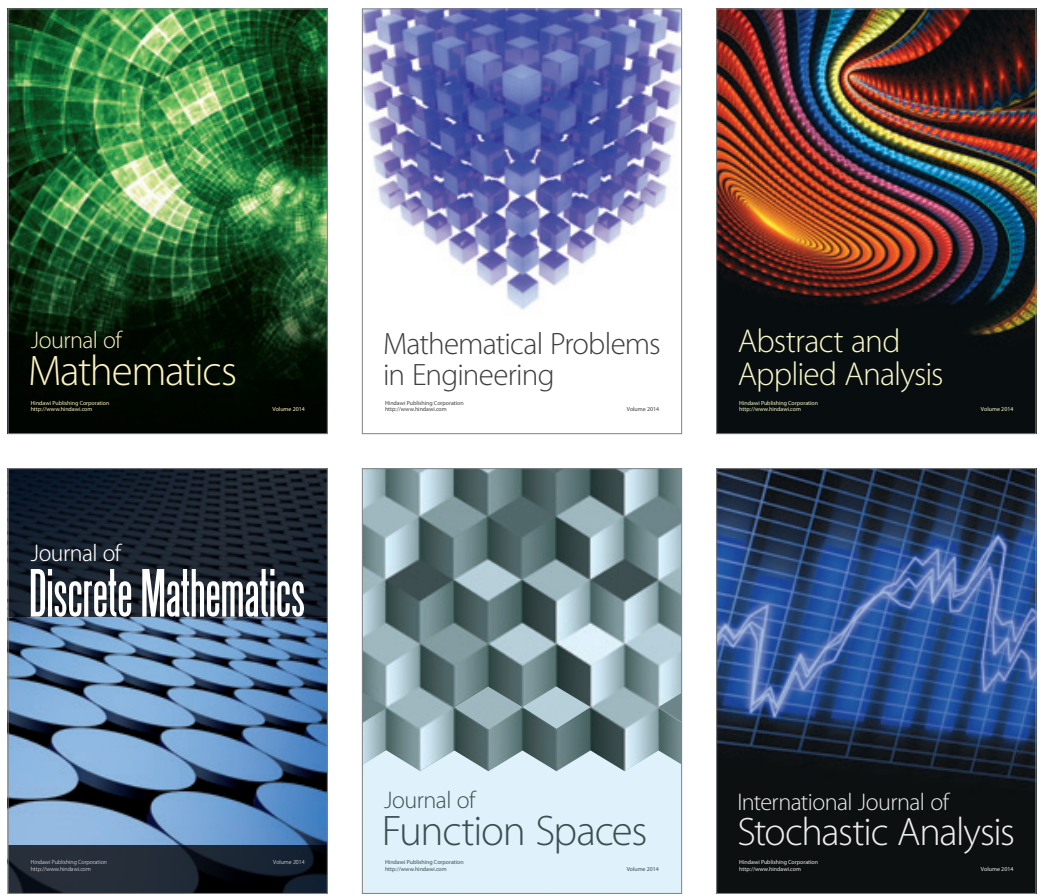

Journal of

Function Spaces

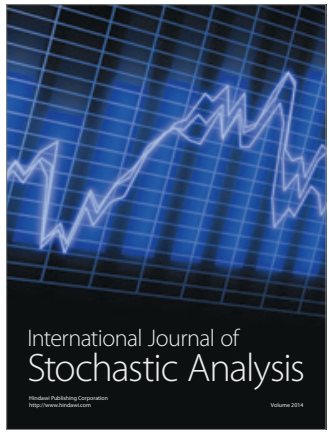

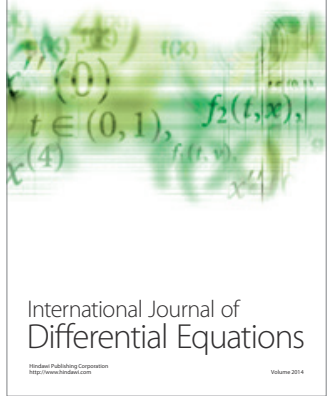
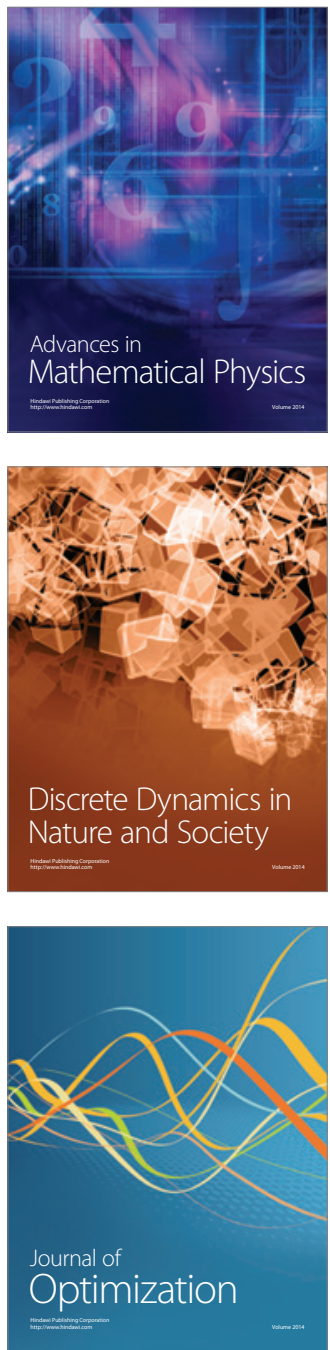